\title{
Synthesis of new 7-azabicyclo[2.2.1]heptane derivatives
}

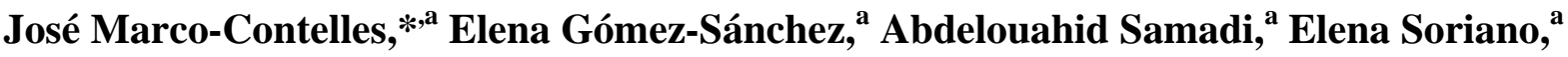 \\ Carolina Valderas, ${ }^{a}$ Mónica Álvarez-Pérez, ${ }^{a}$ and María do Carmo Carreiras ${ }^{b}$ \\ ${ }^{a}$ Laboratorio de Radicales Libres y Química Computacional, Instituto de Química Orgánica \\ General (CSIC), Juan de la Cierva, 3; 28006-Madrid (Spain) \\ ${ }^{b}$ iMEd.UL, Research Institute for Medicines and Pharmaceutical Sciences, Fac. Pharmacy, \\ Univ. Lisbon, Av. Forças Armadas, 1600-083 Lisbon, Portugal \\ E-mail: iqoc21@iqog.csic.es
}

This manuscript is dedicated to Prof. Benito Alcaide on occasion of his 60th birthday

\begin{abstract}
The synthesis of new 7-azabicyclo[2.2.1] heptane derivatives has been achieved in a four-step synthetic sequence, starting from readily available cyclohex-3-enecarboxylic acid, Curtius reaction, stereoselective bromination leading to major benzyl(cis-3,trans-4-dibromocyclohex-1yl)carbamates (amides or sulfonamides), followed by NaH-mediated intramolecular cyclization. The synthesis and free radical cyclization of precursors 4-7, as well as the synthesis of a conformationally constrained epibatidine analogue 3 exploiting the reactivity of the 7azabicyclo[2.2.1]hept-2-yl radical in intramolecular reactions, are described. The $N$-sulfonyl functional motif is the only one to afford a cyclized product when incorporated in the radical precursor.
\end{abstract}

Keywords: 7-Azabicyclo[2.2.1] heptane derivatives, conformationally constrained epibatidine analogues, $N$-(arylmethyl)cyclohex-3-enamines, 7-azabicyclo[2.2.1]hept-2-yl radical, intramolecular free radical reactions

\section{Introduction}

Epibatidine 1, ${ }^{1}$ an alkaloid isolated from the skin of the Ecuadorian poison frog Epipedobates tricolor, ${ }^{2}$ is a powerful analgesic agent 200 times more potent than morphine, with high affinity for the nicotinic acetylcholine receptor (nAChR). ${ }^{3}$ Epibatidine strongly binds at $\alpha 4 \beta 2$ subtype nAChRs, showing values several times higher than nicotine $(2)^{2}$ (Figure 1). However, the toxic effects associated with this drug have hampered its clinical application. ${ }^{4}$ 


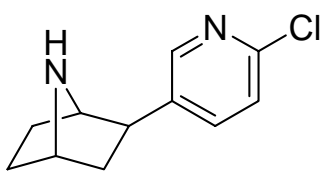

Epibatidine 1

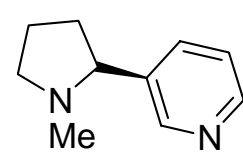

Nicotine 2

Figure 1. Structure of epibatidine 1 and nicotine 2.

In the last years a number of methodologies have been reported for the total synthesis of epibatidine and 7-azabicyclo[2.2.1] heptane derivatives. ${ }^{5 a}$ In the search for epibatidine-type compounds devoid of secondary effects, a number of structure-activity relationship studies have been reported. Thus, great attention has been paid to the synthesis and biological evaluation of epibatidine analogues, ${ }^{5 b}$ either heterocyclic ${ }^{6}$ or conformationally constrained. ${ }^{7}$

We have recently described the synthesis of 7-substituted exo-2-bromo-7azabicyclo[2.2.1] heptane derivatives ${ }^{8,9}$ following a potent method based on a four-step synthetic sequence, starting from readily available cyclohex-3-enecarboxylic acid, Curtius reaction, stereoselective bromination leading to major tert-butyl (or benzyl) (cis-3,trans-4dibromocyclohex-1-yl)carbamates (or 2,2,2-trifluoroacetamides) ${ }^{10}$ followed by NaH-mediated intramolecular cyclization (Scheme 1). ${ }^{11}$

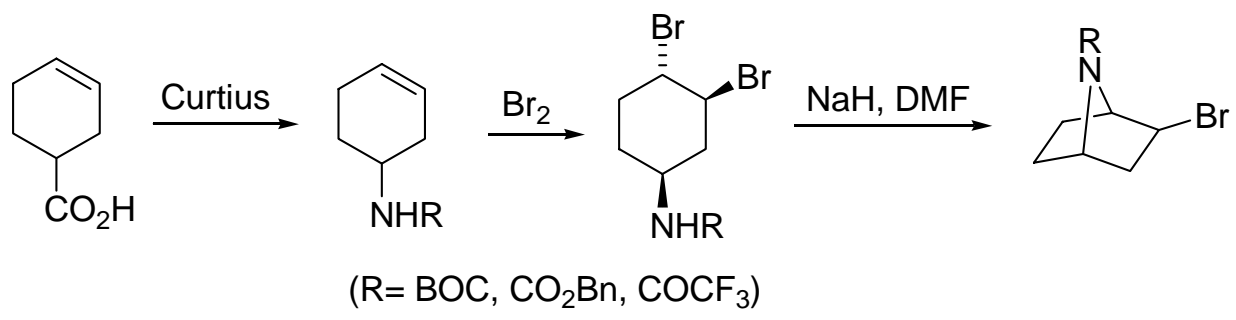

Scheme 1. General approach for the syntheis of 7-azabicyclo[2.2.1] heptane derivatives (ref. 9). 


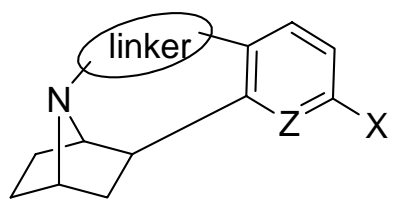

(I)

$(\mathrm{X}=\mathrm{Cl}, \mathrm{H} ; \mathrm{Z}=\mathrm{N}, \mathrm{CH})$

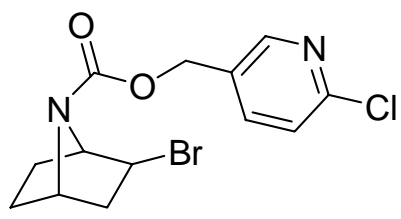

4

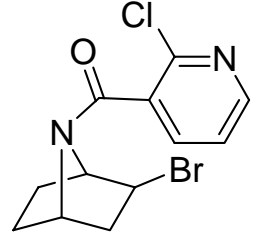

5

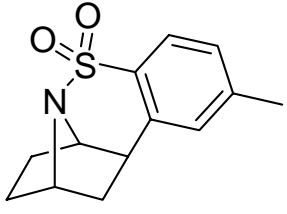

3

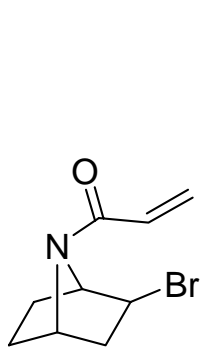

6

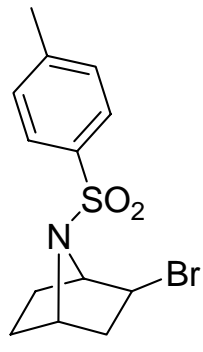

7

Figure 2. Structure of conformationally constrained epibatidine analogues (I), the target molecule 3, and the radical precursors 4-7.

Our current interest in the synthesis and biological evaluation of new epibatidine analogues using intermolecular free radical reactions exploiting the reactivity of the 7azabicyclo[2.2.1] hept-2-yl radical, ${ }^{12}$ prompted us to apply this strategy to prepare conformationally constrained epibatidine analogues of type I (Figure 2). In Scheme 2 we show our general approach for the synthesis of this type of compounds, based on the intramolecular free radical cyclization of species II, readily available from precursors III bearing convenient radical acceptors at C7 and good leaving groups at C2 (Scheme 2).

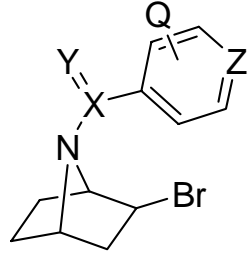

II

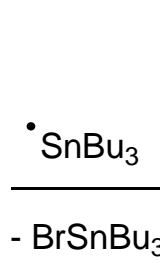

$-\mathrm{BrSnBu}_{3}$

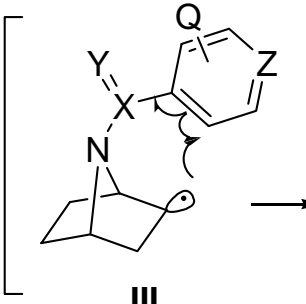

III

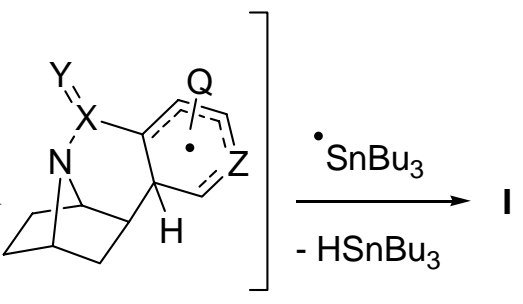

$-\mathrm{HSnBu}_{3}$

Scheme 2. Intramolecular free radical reaction for the preparation odf compounds I.

Although the synthesis and reactivity of 7-norbornenyl, ${ }^{13}$ norborn-5-en-2-yl, ${ }^{14}$ norborn-2$\mathrm{yl}^{15,16}$ radicals is well known, the chemistry of 7-azabicyclo[2.2.1]hept-2-yl radicals has been scarcely investigated. Fraser and Swingle reported the chlorination of 7-trichloroacetyl-7azabicyclo[2.2.1] heptane with sulfuryl chloride in the presence of benzoyl peroxide. ${ }^{17}$ More 
recently, and when this work was in progress, Armstrong and co-workers reported what appears to be the first intramolecular cyclization of a precursor in this series, namely exo-2-bromo-7(toluene-4-sulfonyl)-7-azabicyclo[2.2.1] heptane. ${ }^{18}$

Here we report the synthesis of the conformationally constrained epibatidine analogues 3 , the preparation of radical precursors 4-7 and their intramolecular free radical reactions (Figure 2).

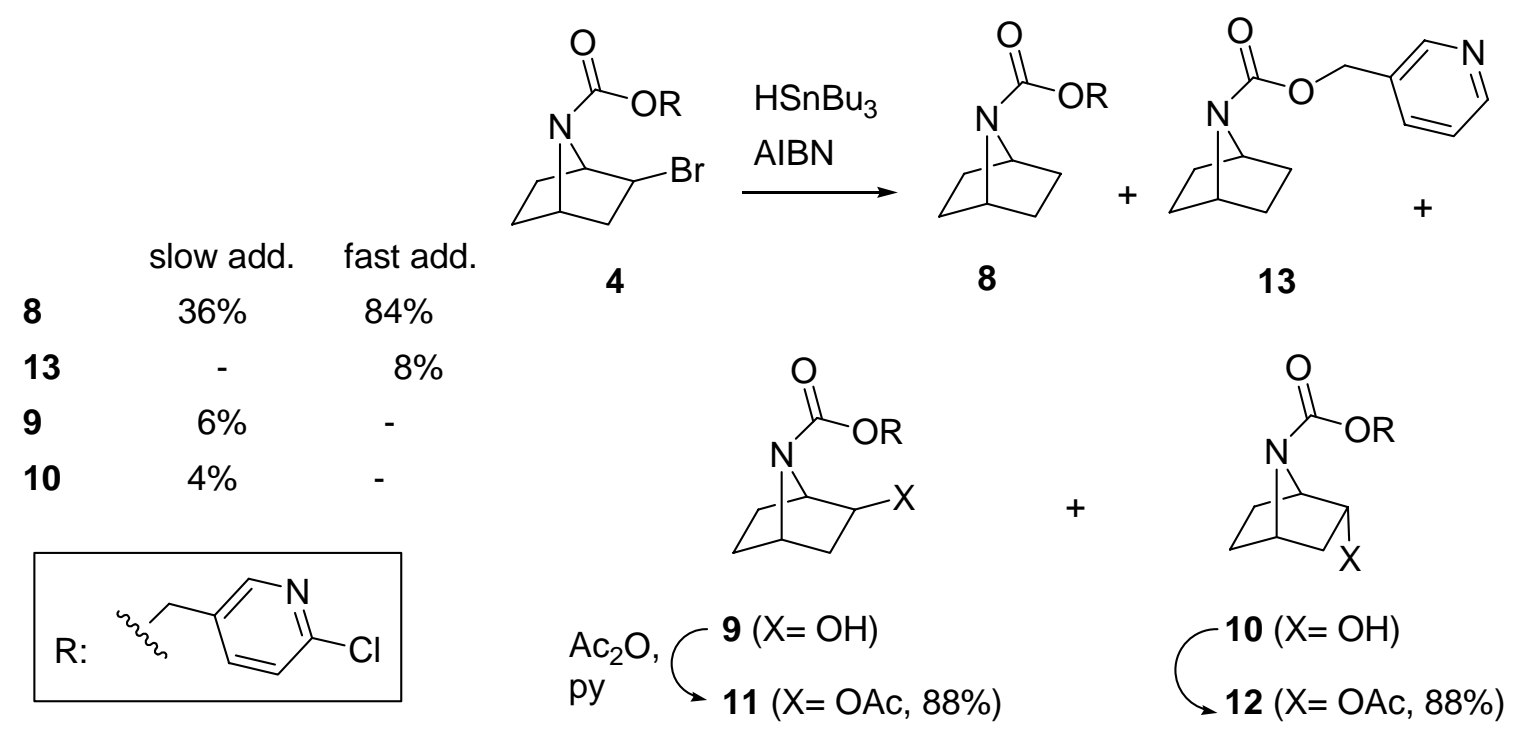

Scheme 3. Free radical cyclization of compound 4.

\section{Results and Discussion}

Under slow addition of $\mathrm{HSnBu}_{3}$, radical precursor $\mathbf{4}^{8,9}$ gave the reduced uncyclized product 8 in 36\% yield, while small amounts of alcohols $\mathbf{9}$ and $\mathbf{1 0}$ (Scheme 3) were also isolated. The structure and relative configuration of the alcohols at $\mathrm{C} 2$ was easily established by NMR spectroscopic analysis on the corresponding acetates 11 and 12. Fast addition of $\mathrm{HSnBu}_{3}$ afforded a higher yield of $\mathbf{8}(84 \%)$, along with $8 \%$ of dechlorinated product $\mathbf{1 3}$ (Scheme 3 ). To sum up, from precursor 4, no cyclized products were detected, regardless of the reaction conditions employed. 

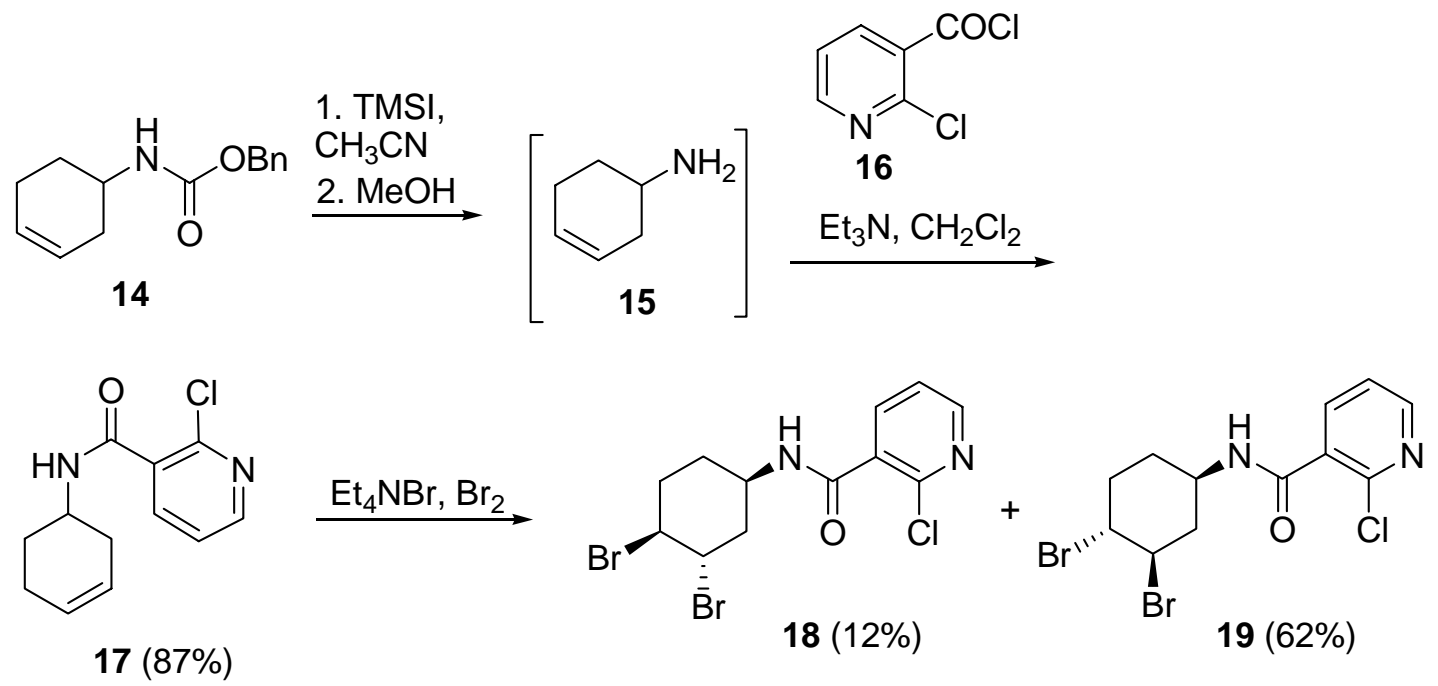

$\mathrm{NaH}, \mathrm{DMF} \downarrow$ (from 19)

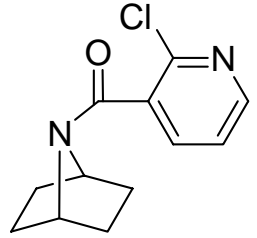

$20(52 \%)$

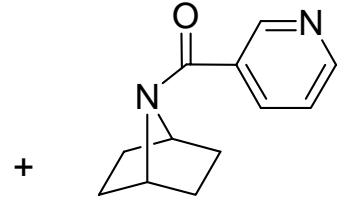

$21(24 \%)$

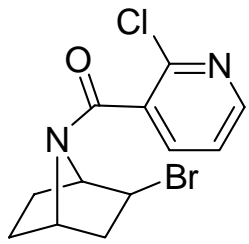

$5(81 \%)$

Scheme 4. Synthesis and free radical cyclization of compound 5.

In order to prepare precursor 5, our first choice was to apply our synthetic sequence ${ }^{9}$ starting with cyclohex-3-enecarboxylic acid, and a Curtius type reaction with 2-chloronicotinic acid. Unfortunately, we were unable to obtain a convenient yield of the desired amide. Consequently, we were forced to use carbamate $14,{ }^{9}$ promote the deprotection reaction, and transform the free, not isolated amine $15^{19}$ into amide $\mathbf{1 7}$ by simple amidation reaction with 2-chloronicotinic acid chloride $\mathbf{1 6}^{20}$ (Scheme 4). As previously reported for analogous amides, bromination ${ }^{11}$ of cyclohex-3-enamide 17 provided a mixture of minor 1,4-cis- and major 1,4-trans-3,4-dibromo derivatives, 18 and 19, that were easily separated, and transformed (only for pure 1,4-trans-3,4dibromo 19) into precursor 5 by reaction with $\mathrm{NaH}$ in $\mathrm{DMF}^{8}$ (Scheme 4). Free radical cyclization of compound 5 under slow addition gave the partially reduced, uncyclized derivative 20, and the fully dehalogenated compound 21 (Scheme 4), as the only reaction products.

Next, precursor 6 was synthesized in good overall yield as shown in Scheme 5, from 3cyclohex-3-enecarboxylic acid 22, via intermediates $24^{21}$ and compound 26 . Unfortunately, the free radical cyclization of bromide $\mathbf{6}$ gave a complex reaction mixture, and no pure defined compound could be isolated and characterized. 


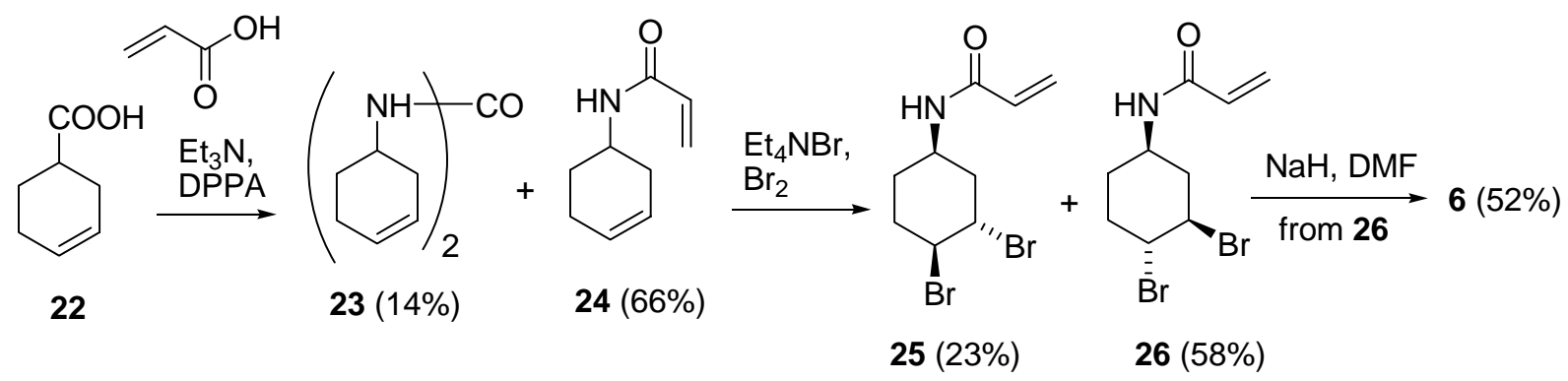

Scheme 5.Synthetic pathway for the preparation of target compound 6.

In retrospect, the success of the Curtius reaction of cyclohex-3-enecarboxylic acid 22 with acrylic acid (Scheme 5), and the failure to accomplish the same reaction with nicotinic acid (see above) was surprising, but we have no rationale for this behaviour. In fact, in this project we were able to carry out the same Curtius reaction with acetic acid to provide acetamide $27,{ }^{22}$ that submitted to the standard protocol afford, via intermediate 29, afforded bromide $\mathbf{3 0}$ (Scheme 6).

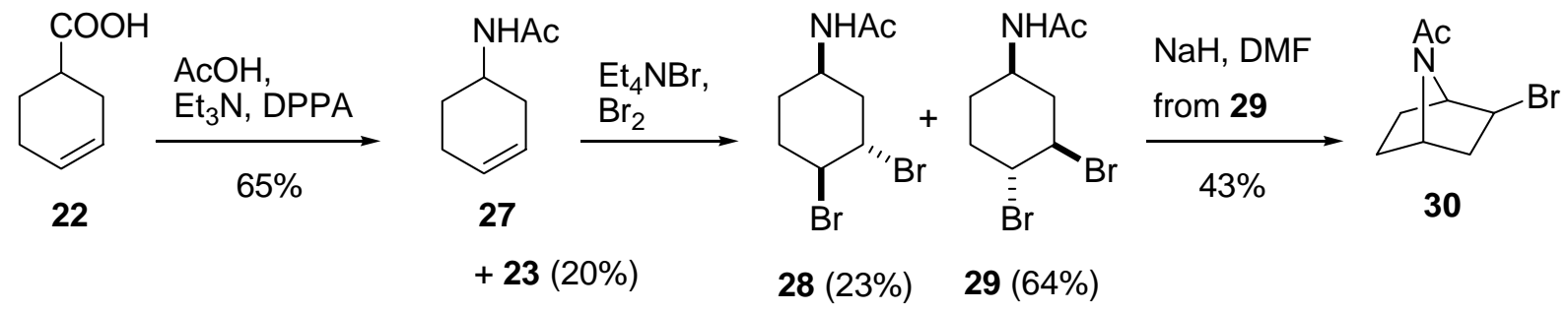

Scheme 6. Synthetic pathway for the preparation of compound 30.

The synthesis of precursor 7 was readily achieved from carbamate $31^{8,9}$ via intermediate $32,{ }^{23}$ obtained after reaction with trifluoroacetic acid, and treatment with $p$-tosyl chloride (Scheme 7). At this point we considered the copper(II)-catalyzed oxidative cyclization of unsaturated sulfonylamides, ${ }^{24}$ but our efforts to achieve a satisfactory result were fruitless, and we turned to our initial plan. Consequently, bromination of compound 32, as usual ${ }^{11}$ gave compounds 33 (70\%) and 34 (20\%). Then, major N-(cis-3-trans-4-dibromocyclohexyl)-4methylbenzenesulfonamide 33, submitted to NaH-mediated cyclization, gave precursor 7 . This compound was identical in its spectroscopic data to the compound reported by Armstrong, ${ }^{18}$ but was prepared in four steps in $41 \%$ overall yield from cyclohex-3-ene carboxylic acid, a stable product, while the previously reported synthesis takes place in four steps also, but in $11 \%$ overall yield from 2-methoxy-3,4-dihydropyran, a flammable product that must be used with caution. ${ }^{25}$ Finally, and as expected and reported, free radical cyclization of precursor $\mathbf{7}$ provided the ring 
closure derivative 3 in 30\% yield, a product which showed analytical and spectroscopic data in good agreement with its structure, and similar to those described. ${ }^{18}$<smiles>CC(C)(C)OC(=O)NC1CC=CCC1</smiles>

31

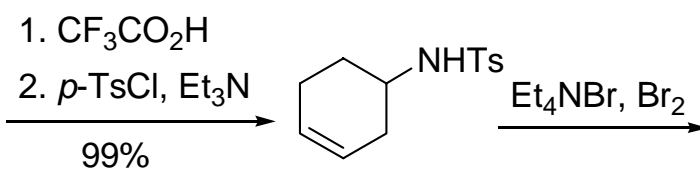

32<smiles>[13CH3][C@H]1CC[C@@H](Br)[C@H](Br)C1</smiles>

$33(70 \%)$

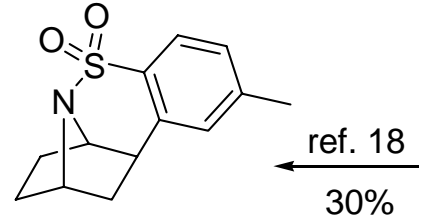

3

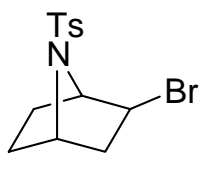

7

Scheme 7. Synthesis and free radical reaction of precursor 7.

\section{Conclusions}

We have reported the synthesis and reactivity of the 7-azabicyclo[2.2.1]hept-2-yl radicals in intramolecular reaction processes, and have shown the scope and limitations of this strategy for the synthesis of conformationally constrained epibatidine analogues. We have detailed the synthesis and free radical cyclization of radical precursors 4-7. In overall, a carbonyl functional group as in a carbamate (precursor 4), or in an amide (precursor 5 and 6), to link the radical trap to the nitrogen at the $\mathrm{C} 7$ position, provides precursors that gave reduced, uncyclized reaction products. Conversely, the $\mathrm{N}$-sulfonyl motif is the only ones able to afford a cyclized product in acceptable chemical yields. As a result we have described here the synthesis of the constrained epibatidine analogue 3 .

\section{Experimental Section}

General Procedures. Melting points were determined on a microscope type apparatus, and are uncorrected. ${ }^{1} \mathrm{H}$ NMR and ${ }^{13} \mathrm{C}$ NMR spectra were recorded at $\mathrm{rt}$ in $\mathrm{CDCl}_{3}$, at 300, 400 or 500 $\mathrm{MHz}$ and at 75, 100 or $125 \mathrm{MHz}$, respectively, using solvent peaks $\left(\mathrm{CDCl}_{3}\right.$ : $7.27(D), 77.2(C)$ ppm; $\mathrm{D}_{2} \mathrm{O}: 4.60 \mathrm{ppm}$ ) as internal reference. The assignment of chemical shifts is based on standard NMR experiments $\left({ }^{1} \mathrm{H},{ }^{13} \mathrm{C}\right.$-DEPT, ${ }^{1} \mathrm{H},{ }^{1} \mathrm{H}-\mathrm{COSY}$, gHSQC, gHMBC). In the NMR 
spectra values with $(*)$ can be interchanged. Values with (') show the invertomers, when distinguishable. Mass spectra were recorded on a GC/MS spectrometer with an API-ES ionization source. Elemental analyses were performed at CQO (CSIC, Spain). TLC was performed on silica F254 and detection by UV light at $254 \mathrm{~nm}$ or by charring with either ninhydrin, anisaldehyde or phosphomolybdic- $\mathrm{H}_{2} \mathrm{SO}_{4}$ dyeing reagents. Where anhydrous solvents were needed, they were purified following the usual procedures. In particular, dry DMF was critical for the outcome of the cyclization reaction, and was either distilled at reduced pressure or bought from commercial sources. Column chromatography was performed on silica gel 60 (230 mesh).

\section{Synthesis and intramolecular free radical reaction of precursor 4. A. Slow addition of $\mathrm{HSnBu}_{3}$}

To a deoxygenated solution of carbamate $4^{8,9}(58 \mathrm{mg}, 0.17 \mathrm{mmol})$ in dry toluene $(8 \mathrm{~mL}, 0.02 \mathrm{M})$ and AIBN $(5 \mathrm{mg}), \mathrm{HSnBu}_{3}(0.07 \mathrm{~mL}, 0.25 \mathrm{mmol}, 1.5$ equiv) in dry, deoxygenated toluene $(2 \mathrm{~mL})$ containing AIBN $(5 \mathrm{mg})$ was slowly added in $14 \mathrm{~h}$ at $95{ }^{\circ} \mathrm{C}$. After the addition the mixture was heated at the same temperature for $10 \mathrm{~h}$ more. The reaction was cooled at $\mathrm{rt}$, the solvent was removed, the residue was dissolved in ethyl ether, and washed with an aqueous saturated KF solution. The organic phase was dried over $\mathrm{Na}_{2} \mathrm{SO}_{4}$, filtered, and the solvent was evaporated. The crude was submitted to chromatography $(20 \% \rightarrow 50 \%$ hexane: AcOEt), to give compound 8 (16 mg, 36\%), a mixture of compounds $4 / 8$ (11 mg, 4:1) and alcohols 9 (2.6 mg, 6\%) and 10 ( $2 \mathrm{mg}, 4 \%)$. 8: white solid; $\mathrm{mp} \mathrm{87-9}{ }^{\circ} \mathrm{C}$; IR (KBr) $\vee 3043,2955,1705,1460,1154$ $\mathrm{cm}^{-1} ;{ }^{1} \mathrm{H}$ NMR $\left(\mathrm{CDCl}_{3}, 300 \mathrm{MHz}\right) \delta 8.39\left(\mathrm{~d}, J=2.4 \mathrm{~Hz}, 1 \mathrm{H}, \mathrm{H2}{ }^{\prime}\right), 7.67$ (dd, $J=2.4,8.1 \mathrm{~Hz}, 1 \mathrm{H}$, H4'), 7.32 (d, J= 8.1 Hz, 1H, H5'), 5.09 (s, 2H, $\mathrm{CH}_{2} \mathrm{O}$ ), 4.28 (s, 2H, H1, H4), 1.81-1.70 (m, 4H), 1.48-1.38 (m, 4H); ${ }^{13} \mathrm{C}^{\mathrm{NMR}}\left(\mathrm{CDCl}_{3}, 100 \mathrm{MHz}\right) \delta 154.9$ (NCOO), 151.2 (C6'), $149.4\left(\mathrm{C}^{\prime}\right)$, 138.8 (C4'), 131.6 (C3'), $124.3\left(\mathrm{C}^{\prime}\right.$ '), $63.4\left(\mathrm{CH}_{2} \mathrm{O}\right), 56.4(\mathrm{C} 1, \mathrm{C} 4), 29.8\left(4 \mathrm{xCH}_{2}\right)$; MS (ES) $\mathrm{m} / \mathrm{z}$ $[\mathrm{M}+1]^{+} \quad 267.3 / 269.2, \quad[\mathrm{M}+23]^{+}$289.2/291.3, $[2 \mathrm{M}+23]^{+}$555.5/557.5. Anal. Calcd. for $\mathrm{C}_{13} \mathrm{H}_{15} \mathrm{ClN}_{2} \mathrm{O}_{2}$ : C, 58.54; H, 5.67; N, 10.50. Found: C, 58.42; H, 5.60; N, 10.75 .

(6-Chloropyridin-3-yl)methyl exo-2-hydroxy-7-azabicyclo[2.2.1]heptane-7-carboxylate 9. ${ }^{1} \mathrm{H}$ NMR $\left(\mathrm{CDCl}_{3}, 300 \mathrm{MHz}\right) \delta 8.41(\mathrm{~d}, J=2.2 \mathrm{~Hz}, 1 \mathrm{H}), 7.69(\mathrm{dd}, J=2.5,8.2 \mathrm{~Hz}, 1 \mathrm{H}), 7.33(\mathrm{~d}, J=$ $8.2 \mathrm{~Hz}, 1 \mathrm{H}), 5.12(\mathrm{~s}, 2 \mathrm{H}), 4.34(\mathrm{t}, J=4.7 \mathrm{~Hz}, 1 \mathrm{H}), 4.20(\mathrm{~d}, J=4.8 \mathrm{~Hz}, 1 \mathrm{H}), 3.95(\mathrm{td}, J=1.8,6.7$ $\mathrm{Hz}, 1 \mathrm{H}), 1.88(\mathrm{dd}, J=6.8,13.1 \mathrm{~Hz}, 1 \mathrm{H}), 1.82-1.54(\mathrm{~m}, 4 \mathrm{H}), 1.36-1.24(\mathrm{~m}, 2 \mathrm{H}) ; \mathrm{MS}(\mathrm{ES}) \mathrm{m} / \mathrm{z}$ $[\mathrm{M}+1]^{+}$283.0/285.0, [M+23 $]^{+}$305.0/307.0.

(6-Chloropyridin-3-yl)methyl endo-2-hydroxy-7-azabicyclo[2.2.1]heptane-7-carboxylate 10. ${ }^{1} \mathrm{H} \mathrm{NMR}\left(\mathrm{CDCl}_{3}, 300 \mathrm{MHz}\right) \delta 8.40(\mathrm{~d}, J=2.2 \mathrm{~Hz}, 1 \mathrm{H}), 7.68(\mathrm{dd}, J=2.5,8.2 \mathrm{~Hz}, 1 \mathrm{H}), 7.34(\mathrm{~d}, J=$ $8.0 \mathrm{~Hz}, 1 \mathrm{H}), 5.10(\mathrm{~s}, 2 \mathrm{H}), 4.42-4.31(\mathrm{~m}, 1 \mathrm{H}), 4.28-4.18(\mathrm{~m}, 2 \mathrm{H}), 2.30-2.15(\mathrm{~m}, 2 \mathrm{H}), 1.88-1.72$ (m, 1H), 1.72-1.50 (m, 3H), $1.11(\mathrm{dd}, J=3.4,12.7 \mathrm{~Hz}, 1 \mathrm{H})$; MS (ES) $\mathrm{m} / \mathrm{z}[\mathrm{M}+1]^{+}$283.0/285.0, $[\mathrm{M}+23]^{+}$305.0/307.0. 


\section{Acetylation of alcohols 9 and 10. General Method}

The alcohols were treated with a mixture of $\mathrm{Ac}_{2} \mathrm{O} / \mathrm{py}(1: 1$, vol), at $\mathrm{rt}$ for $21 \mathrm{~h}$. Then, the solvents and reagents were evaporated, and the residue was submitted to chromatography (25\% hexane: AcOEt) to give the expected compounds.

(6-Chloropyridin-3-yl)methyl exo-2-acetoxy-7-azabicyclo[2.2.1]heptane-7-carboxylate (11). Following the General Method for acetylation compound $9(14.9 \mathrm{mg}, 0.05 \mathrm{mmol})$ was treated with $\mathrm{Ac}_{2} \mathrm{O} /$ py $(2 \mathrm{~mL}, 2 \mathrm{~mL})$ to give compound 11 (15 mg, 88\%): oil; IR (film) v 3086, 2955, 1738, 1709, 1590, 1568, 1462, 1409, 1377, 1317, 1243, $1099 \mathrm{~cm}^{-1} ;{ }^{1} \mathrm{H} \mathrm{NMR}\left(\mathrm{CDCl}_{3}, 300 \mathrm{MHz}\right)$ $\delta 8.40$ (d, $J=2.1 \mathrm{~Hz}, 1 \mathrm{H}, \mathrm{H} 2$ '), 7.68 (dd, $J=2.4,8.2 \mathrm{~Hz}, 1 \mathrm{H}, \mathrm{H} 4$ '), 7.33 (d, J=8.2 Hz, 1H, H5'), $5.11\left(\mathrm{~s}, 2 \mathrm{H}, \mathrm{CH}_{2} \mathrm{O}\right), 4.73$ (dd, $\left.J=2.4,7.0 \mathrm{~Hz}, 1 \mathrm{H}, \mathrm{H} 2\right), 4.42-4.30$ (m, 2H, H1, H4), 2.03-1.87 (m, $4 \mathrm{H}, \mathrm{CH}_{3}, \mathrm{H} 3_{\text {endo }}$ ), 1.87-1.61 (m, 3H, H3 exo $_{1} \mathrm{H} 5_{\text {exo }}, \mathrm{H} 6_{\text {exo }}$ ), 1.47-1.29 (m, $2 \mathrm{H}, \mathrm{H} 5_{\text {endo }}, \mathrm{H} 6_{\text {endo }}$ ); ${ }^{13} \mathrm{C}$ NMR (CDCl 3 , 75 MHz) $\delta 170.9$ (COO), 155.1 (NCOO), 151.4 (C6'), 149.5 (C2'), 138.8 (C4'), 131.5 (C3'), 124.3 (C5'), 76.5 (C2), $63.6\left(\mathrm{CH}_{2} \mathrm{O}\right), 60.5,55.4$ (C1, C4), 39.0 (C3), 28.5, 24.7 (C5, C6), $21.2\left(\mathrm{CH}_{3}\right) ; \mathrm{MS}(\mathrm{ES}) \mathrm{m} / \mathrm{z}[\mathrm{M}+1]^{+}$325.3/327.2, $[\mathrm{M}+23]^{+}$347.2/349.2. Anal. Calcd. for $\mathrm{C}_{15} \mathrm{H}_{17} \mathrm{ClN}_{2} \mathrm{O}_{4}$ : C, 55.48; H, 5.28; N, 8.63. Found: C, 55.76; H, 5.19; N, 8.71.

(6-Chloropyridin-3-yl)methyl endo-2-acetoxy-7-azabicyclo[2.2.1]heptane-7-carboxylate (12). Following the General Method for acetylation compound 10 (6.8 $\mathrm{mg}, 0.02 \mathrm{mmol}$ ) was treated with $\mathrm{Ac}_{2} \mathrm{O} /$ py $(2 \mathrm{~mL}, 2 \mathrm{~mL})$ to give compound 12 (6.9 mg, 88\%): white solid; mp 92$4{ }^{\circ} \mathrm{C}$; IR $(\mathrm{KBr}) \vee 3023,2963,1732,1704,1590,1571,1465,1412,1376,1308,1264,1240,1116$ $\mathrm{cm}^{-1} ;{ }^{1} \mathrm{H}$ NMR $\left(\mathrm{CDCl}_{3}, 400 \mathrm{MHz}\right) \delta 8.40(\mathrm{dd}, J=0.8,2.3 \mathrm{~Hz}, 1 \mathrm{H}, \mathrm{H} 2$ ') $7.68(\mathrm{dd}, J=2.7,8.2 \mathrm{~Hz}$, 1H, H4'), 7.34 (dd, J=0.8, 8.2 Hz, 1H, H5'), 5.10 (s, 2H, $\mathrm{CH}_{2} \mathrm{O}$ ), 4.99-4.90 (m, 1H, H2), 4.48 (t, $J=4.7 \mathrm{~Hz}, 1 \mathrm{H}, \mathrm{H} 1), 4.31-4.24(\mathrm{~m}, 1 \mathrm{H}, \mathrm{H} 4), 2.54-2.25$ (m, 1H, H3 exo), 2.07 (s, 3H, $\left.\mathrm{CH}_{3}\right), 1.96$ (ddd, $J=3.9,9.0,12.7 \mathrm{~Hz}, 1 \mathrm{H}, \mathrm{H} 6_{\text {endo }}$ ), 1.87-1.75 (m, 1H, H5 exo $_{\text {) }}, 1.71-1.59$ (m, 1H, H6 $6_{\text {exo }}$ ), 1.54 (ddd, $\left.J=4.3,9.0,11.7 \mathrm{~Hz}, 1 \mathrm{H}, \mathrm{H} 5_{\text {endo }}\right), 1.24$ (dd, $\left.J=3.5,13.3 \mathrm{~Hz}, 1 \mathrm{H}, \mathrm{H} 3_{\text {endo }}\right) ;{ }^{13} \mathrm{C} \mathrm{NMR}\left(\mathrm{CDCl}_{3}\right.$, $100 \mathrm{MHz}) \delta 170.8$ (COO), 154.8 (NCOO), 151.5 (C6'), 149.6 (C2'), 138.9 (C4'), 131.3 (C3'), $124.4\left(\mathrm{C}^{\prime}\right.$ '), $72.7(\mathrm{C} 2), 63.7\left(\mathrm{CH}_{2} \mathrm{O}\right), 58.2$ (C1), 57.0 (C4), 37.0 (C3), 29.4 (C5), 22.2 (C6), 21.1 $\left(\mathrm{CH}_{3}\right)$; MS (ES) $\mathrm{m} / \mathrm{z}[\mathrm{M}+1]^{+} 325.2 / 327.2$, $[\mathrm{M}+23]^{+}$347.2/349.2. Anal. Calcd. for $\mathrm{C}_{15} \mathrm{H}_{17} \mathrm{ClN}_{2} \mathrm{O}_{4}$ : C, 55.48; H, 5.28; N, 8.63. Found: C, 55.76; H, 5.16; N, 8.91.

B. Fast addition of $\mathbf{H S n B u}_{3}$. $\mathrm{HSnBu}_{3}(0.07 \mathrm{~mL}, 0.25 \mathrm{mmmol}, 1.7$ equiv $)$ in dry toluene $(0.5 \mathrm{~mL})$ was added to compound $4(52 \mathrm{mg}, 0.15 \mathrm{mmol})$ in toluene $(0.02 \mathrm{M})$ under reflux. After addition the mixture was heated at the same temperature for $3 \mathrm{~h}$. The reaction was cooled at $\mathrm{rt}$, the solvent was removed, and the residue was dissolved in ethyl ether and washed with an aqueous saturated KF solution. The organic phase was dried over $\mathrm{Na}_{2} \mathrm{SO}_{4}$, filtered and the solvent evaporated. The crude was submitted to chromatography $(20 \% \rightarrow 50 \%$ hexane: AcOEt) to yield 6-chloropyridin-3-yl) methyl-7-azabicyclo[2.2.1]heptane-7-carboxylate 8 (34 $\mathrm{mg}$, 84\%) and pyridin-3-ylmethyl 7-azabicyclo[2.2.1]heptane-7-carboxylate 13 (2.6 mg, 8\%). 13: oil; IR (film) v 2950, 1705, $1429 \mathrm{~cm}^{-1} ;{ }^{1} \mathrm{H} \mathrm{NMR}\left(\mathrm{CDCl}_{3}, 500 \mathrm{MHz}\right) \delta 8.63(\mathrm{~d}, J=1.7 \mathrm{~Hz}, 1 \mathrm{H}$, H2'), 8.57 (dd, $J=4.8$ Hz, 1H, H6'), 7.70 (dm, $J=7.8$ Hz, 1H, H4'), 7.30 (dd, $J=4.8,7.7$ Hz, 1H, $\mathrm{H}^{\prime}$ '), 5.13 (s, 2H, $\left.\mathrm{CH}_{2} \mathrm{O}\right), 4.30(\mathrm{~s}, 2 \mathrm{H}, \mathrm{H} 1, \mathrm{H} 4), 1.84-1.71(\mathrm{~m}, 4 \mathrm{H}), 1.50-1.35(\mathrm{~m}, 4 \mathrm{H})$; ${ }^{13} \mathrm{C} \mathrm{NMR}$ $\left(\mathrm{CDCl}_{3}, 125 \mathrm{MHz}\right) \delta 155.2$ (c, NCOO), 149.6 (C2'), 149.5 (C6'), 135.9 (C4'), 132.7 (C3'), 123.6 
(C5'), $64.3\left(\mathrm{CH}_{2} \mathrm{O}\right), 56.4(\mathrm{C} 1, \mathrm{C} 4), 29.9$ (br, C2, C3, C5, C6); MS (ES) m/z [M+1] 233.1, $[\mathrm{M}+23]^{+}$255.1. HRMS calcd for $\mathrm{C}_{13} \mathrm{H}_{17} \mathrm{~N}_{2} \mathrm{O}_{2} 233.1284\left(\mathrm{M}+\mathrm{H}^{+}\right)$, found $233.1283\left(\mathrm{M}+\mathrm{H}^{+}\right)$.

Synthesis and intramolecular free radical reaction of precursor 5. 2-Chloro- $\mathrm{N}$-(cyclohex-3enyl)nicotinamide (17)

To a solution of carbamate $14^{9}(795 \mathrm{mg}, 3.44 \mathrm{mmol})$ in dry $\mathrm{CH}_{3} \mathrm{CN}$ (44 mL, $\left.0.1 \mathrm{M}\right)$, TMSI ( $1.96 \mathrm{~mL}, 13.33 \mathrm{mmol}, 3.88$ equiv) was added at $0{ }^{\circ} \mathrm{C}$, and the mixture was stirred for $30 \mathrm{~min}$ under argon. Then, methanol $(12.3 \mathrm{~mL})$ was added, and the reaction was warmed at $\mathrm{rt}$. After 30 min, the solvent was removed, and resulting cyclohex-3-enamine $15^{19}$ was dissolved in dry $\mathrm{CH}_{2} \mathrm{Cl}_{2}$ (14 mL, $0.2 \mathrm{M}$ ). The solution was cooled at $0{ }^{\circ} \mathrm{C}, \mathrm{Et}_{3} \mathrm{~N}(1.20 \mathrm{~mL}, 8.62 \mathrm{mmol}, 2.51 \mathrm{equiv})$ and acid chloride 16 (761 mg, $4.31 \mathrm{mmol}, 1.26$ equiv, prepared from commercial 2chloronicotinic acid as described $)^{20}$ was added. The bath was removed and the reaction was warmed at $\mathrm{rt}$ for $17 \mathrm{~h}$. Then, $\mathrm{H}_{2} \mathrm{SO}_{4}(8.8 \mathrm{~mL}, 2 \mathrm{M})$ was added. The resulting solution was neutralized with aqueous saturated $\mathrm{NaHCO}_{3}$ solution, and the aqueous layer was extracted with $\mathrm{CH}_{2} \mathrm{Cl}_{2}$ (x3). The organic phases were dried over $\mathrm{MgSO}_{4}$, filtered, and evaporated. The crude was submitted to chromatography (50\% hexane: AcOEt), which gave

2-Chloro- $\boldsymbol{N}$-(cyclohex-3-enyl)nicotinamide (17). (707 mg, 87\%): white solid; 92-94 ${ }^{\circ} \mathrm{C}$; IR $(\mathrm{KBr}) \vee 3470,3268,3062,2920,1635,1585,1543,1397 \mathrm{~cm}^{-1} ;{ }^{1} \mathrm{H}$ NMR $\left(\mathrm{CDCl}_{3}, 400 \mathrm{MHz}\right)$ $\delta 8.46$ (dd, $J=2.0,4.7 \mathrm{~Hz}, 1 \mathrm{H}, \mathrm{H} 6$ '), 8.12 (dd, $J=2.0,7.8 \mathrm{~Hz}, 1 \mathrm{H}, \mathrm{H} 4$ '), 7.35 (dd, J= 4.7, $7.4 \mathrm{~Hz}$, 1H, H5'), 6.52 (br s, 1H, NH), 5.79-5.73 (m, 1H, H4), 5.70-5.63 (m, 1H, H3), 4.44-4.33 (m, 1H, $\mathrm{H} 1), 2.52\left(\mathrm{dm}, J=17.2 \mathrm{~Hz}, 1 \mathrm{H}, \mathrm{H} 2_{\mathrm{eq}}\right), 2.30-2.13$ (m, 2H, 2xH5), 2.13-1.93 (m, 2H, H2 $\left.2_{\mathrm{ax}}, \mathrm{H} 6_{\mathrm{eq}}\right)$, 1.85-1.74 (m, 1H, H6 $\left.{ }_{\mathrm{ax}}\right) ;{ }^{13} \mathrm{C} \mathrm{NMR}\left(\mathrm{CDCl}_{3}, 100 \mathrm{MHz}\right) \oint 164.1$ (NHCO), 150.1 (C6'), 147.2 (C2'), 140.0 (C4'), 131.6 (C3'), 127.4 (C4), 124.2 (C3), 123.0 (C5'), 45.5 (C1), 31.4 (C2), 27.6 (C6), 23.2 (C5); MS (ES) $\mathrm{m} / \mathrm{z}[\mathrm{M}+1]^{+}$237.1/239.1, [M+23] 259.0/261.0. Anal. Calcd. for $\mathrm{C}_{12} \mathrm{H}_{13} \mathrm{ClN}_{2} \mathrm{O}$ : C, 60.89; H, 5.54; N, 11.84. Found: C, 60.68; H, 5.83; N, 11.55.

Bromination of 2-chloro- $\mathbf{N}$-(cyclohex-3-enyl)nicotinamide 17. To a solution of compound $\mathbf{1 7}$ (506 mg, $2.14 \mathrm{mmol}$ ) in dry $\mathrm{CH}_{2} \mathrm{Cl}_{2}(25 \mathrm{~mL}, 0.09 \mathrm{M}), \mathrm{Et}_{4} \mathrm{NBr}(4.51 \mathrm{~g}, 21.4 \mathrm{mmol}, 10$ equiv) was added and the mixture was stirred $5 \mathrm{~min}$ at $\mathrm{rt}$, under argon. Then, $\mathrm{Br}_{2}(0.12 \mathrm{~mL}, 2.35 \mathrm{mmol}, 1.1$ equiv) was added at $-78{ }^{\circ} \mathrm{C}$. After $1 \mathrm{~h}$ stirring, the mixture was warmed at $\mathrm{rt}$, and an aqueous saturated $\mathrm{Na}_{2} \mathrm{~S}_{2} \mathrm{O}_{5}$ solution was added until the color disappeared. The mass was extracted with AcOEt (x3), and the organic phase was dried over $\mathrm{Na}_{2} \mathrm{SO}_{4}$, filtered, and evaporated. The crude was submitted to chromatography (silica gel, $0.2 \% \mathrm{CH}_{2} \mathrm{Cl}_{2}: \mathrm{MeOH}$ ) affording 2-chloro- $N$ (trans-3,cis-4-dibromocyclohexyl)nicotinamide 18 (102 mg, 12\%) and 2-chloro- $N$-(cis-3,trans4-dibromocyclohexyl]nicotinamide 19 (530 mg, 62\%). 18: white solid; 164-6 ${ }^{\circ} \mathrm{C}$; IR (KBr) v 3270, 3071, 2950, 1644, 1544, $1400 \mathrm{~cm}^{-1} ;{ }^{1} \mathrm{H} \mathrm{NMR}\left(\mathrm{CDCl}_{3}, 300 \mathrm{MHz}\right) \delta 8.46(\mathrm{dd}, J=2.0,4.7$ Hz, 1H, H6'), 8.08 (dd, J=2.0, 7.6 Hz, 1H, H4'), 7.35 (dd, J=4.8, 7.6 Hz, 1H, H5'), 6.43 (d, $J \approx$ $7.1 \mathrm{~Hz}, 1 \mathrm{H}, \mathrm{NH}), 4.73-4.50$ (m, 3H, H1, H3, H4), 2.75-2.58 (m, 1H, H5 ax ), 2.48 (ddd, J= 3.2, 11.2, 14.3 Hz, 1H, H2 ax), 2.35 (dm, J=14.3 Hz, 1H, H2 $2_{\text {eq }}$ ), 2.15-2.00 (m, 2H, H5 eq H6 $6_{\text {ax }}$ ), 2.00$1.82\left(\mathrm{~m}, 1 \mathrm{H}, \mathrm{H6}_{\mathrm{eq}}\right) ;{ }^{13} \mathrm{C} \mathrm{NMR}\left(\mathrm{CDCl}_{3}, 75 \mathrm{MHz}\right) \oint 164.1$ (NCO), 151.2 (C6'), 147.2 (C2'), 139.9 (C4'), 131.3 (C3'), 123.0 (C5'), 52.0, 51.3 (C3, C4), 45.0 (C1), 34.6 (C2*), 28.3 (C5*), 27.1 
(C6*); MS (ES) m/z [M+1] 395.0/397.0/398.9. Anal. Calcd. for $\mathrm{C}_{12} \mathrm{H}_{13} \mathrm{Br}_{2} \mathrm{ClN}_{2} \mathrm{O}$ : C, 36.35; $\mathrm{H}$, 3.30; N, 7.07. Found: C, 36.34; H, 3.27; N, 7.04. 19: white solid; $161-3{ }^{\circ} \mathrm{C}$; IR (KBr) $\vee 3436$, 3263, 3071, 2934, 1638, 1551, $1398 \mathrm{~cm}^{-1} ;{ }^{1} \mathrm{H}$ NMR $\left(\mathrm{CDCl}_{3}, 300 \mathrm{MHz}\right) \delta 8.48(\mathrm{dd}, J=2.0,4.8$ Hz, 1H, H6'), 8.09 (dd, J=2.0, 7.6 Hz, 1H, H4'), 7.35 (dd, J=4.8, 7.6 Hz, 1H, H5'), 6.76 (br s, 1H, NH), 4.34-4.16 (m, 3H, H1, H3, H4), 2.91 (dtd, $J=1.9,4.1,13.8 \mathrm{~Hz}, 1 \mathrm{H}, \mathrm{H} 5_{\mathrm{ax}}$ ), 2.58 (ddt, $J=$ $\left.3.7,6.1,14.4 \mathrm{~Hz}, 1 \mathrm{H}, \mathrm{H} 2_{\mathrm{ax}}\right), 2.26-2.12\left(\mathrm{~m}, 1 \mathrm{H}, \mathrm{H} 2_{\mathrm{eq}}\right), 2.11-1.94\left(\mathrm{~m}, 2 \mathrm{H}, \mathrm{H} 5_{\mathrm{eq}}, \mathrm{H} 6_{\mathrm{eq}}\right), 1.68-1.53$

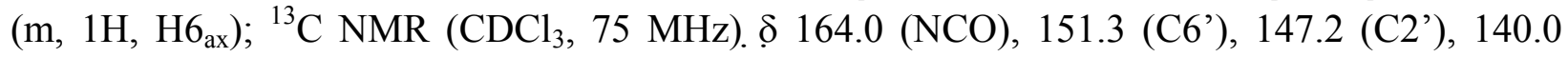
(C4'), 131.3 (C3'), 123.0 (C5'), 54.5, 52.6, 47.2 (C3, C4, C1), 40.3, 32.6, 30.5; EM (ES) m/z $[\mathrm{M}+1]^{+}$395.0/397.0/399.0. Anal. Calcd. for $\mathrm{C}_{12} \mathrm{H}_{13} \mathrm{Br}_{2} \mathrm{ClN}_{2} \mathrm{O}$ : C, 36.35; H, 3.30; N, 7.07. Found: C, 36.51; H, 3.32; N, 6.89.

(2-Bromo-7-azabicyclo[2.2.1]heptan-7-yl)(2-chloropyridin-3-yl)methanone (5). To a solution of compound $19(117 \mathrm{mg}, 0.30 \mathrm{mmol})$ in dry DMF (3 mL, $0.1 \mathrm{M})$ under argon and at $0{ }^{\circ} \mathrm{C}, \mathrm{NaH}$ (15 mg, $0.38 \mathrm{mmol}, 1.27$ equiv, 60\% in oil) was added. The mixture was stirred at $\mathrm{rt}$ for $24 \mathrm{~h}$. Then, the flask was cooled, water was added, the mixture was extracted with ethyl ether (x4) and the organic layer was washed with brine. The organic phase was dried over $\mathrm{MgSO}_{4}$, filtered and evaporated. The crude was submitted to column chromatography (silica gel, $1 \% \mathrm{CH}_{2} \mathrm{Cl}_{2}: \mathrm{MeOH}$ ) to yield radical precursor 5 (76 mg, 81\%): white solid 173-6 ${ }^{\circ} \mathrm{C}$; IR $(\mathrm{KBr}) \vee 3062,2950,1634$, 1579, 1459, 1432, $1400 \mathrm{~cm}^{-1}$; ${ }^{1} \mathrm{H} \mathrm{NMR}\left(\mathrm{CDCl}_{3}, 300 \mathrm{MHz}\right) \delta 8.47-8.46$ (m, H6', minor invertomer), 8.44 (dd, $J=2.0,4.9 \mathrm{~Hz}, \mathrm{H6}$ ', major invertomer), 7.94 (dd, $J=1.9,7.6 \mathrm{~Hz}, \mathrm{H} 4$ ', major invertomer), 7.76 (dd, $J=1.8,7.5 \mathrm{~Hz}, \mathrm{H} 4$ ', minor invertomer), 7.32 (dd, $J=4.8,7.6 \mathrm{~Hz}$, $1 \mathrm{H}, \mathrm{H5}$ '), 5.01 (d, $J=5.4 \mathrm{~Hz}, \mathrm{H} 1$, minor invertomer), 4.94 (t, $J=4.6 \mathrm{~Hz}, \mathrm{H} 4$, major invertomer), 4.15-4.09 (m, H2, minor invertomer), 4.04 (dd, $J=3.4,7.0 \mathrm{~Hz}, \mathrm{H} 2$, major invertomer), 3.88 (d, $J=5.3 \mathrm{~Hz}, \mathrm{H} 1$, major invertomer), 3.82 (t, $J=4.5 \mathrm{~Hz}, \mathrm{H} 4$, minor invertomer), 2.44-2.25 (m, 2H, 2xH3), 2.24-2.06 (m, 1H, H6 exo), 2.06-1.79 (m, 1H, H5 exo), 1.64-1.35 (m, 2H, H5 endo, H6 $6_{\text {endo }}$ ); ${ }^{13} \mathrm{C} \mathrm{NMR}\left(\mathrm{CDCl}_{3}, 75 \mathrm{MHz}\right) \delta 162.8$ (NCO, major invertomer), 162.6 (NCO, minor invertomer), 150.5 (C6'), 147.5 (C2'), 139.2 (C4', major invertomer), 137.4 (C4', minor invertomer), 132.5 (C3', minor invertomer), 132.1 (C3', major invertomer), 122.7 (C5', minor invertomer), 122.5 (C5', major invertomer), 65.4/65.3 (C1, major invertomer; presence of rotamers), 61.5/61.4 (C1, minor invertomer; presence of rotamers), 57.7 (C4, minor invertomer), 53.8/53.7 (C4, major invertomer), 49.9/49.8 (C2, major invertomer), 47.9 (C2, minor invertomer), 44.2 (C3, minor invertomer), 42.9 (C3, major invertomer), 29.7 (C5*, minor invertomer), 28.6 (C5*, major invertomer), 28.2 (C6*, major invertomer), 27.2 (C6*, minor invertomer); MS (ES) m/z [M+1] 315.0/317.0/319.0, $[\mathrm{M}+23]^{+}$336.9/339.0/341.0. Anal. Calcd. for $\mathrm{C}_{12} \mathrm{H}_{12} \mathrm{BrClN}_{2} \mathrm{O}: \mathrm{C}, 45.67 ; \mathrm{H}$, 3.83 ; N, 8.88. Found: C, 45.38; H, 3.79; N, 8.85.

Intramolecular free radical reaction of precursor 5. To a deoxygenated solution of carbamate 43 (40 mg, $0.13 \mathrm{mmol})$ in dry toluene $(6 \mathrm{~mL}, 0.02 \mathrm{M})$, AIBN (2 mg) was added. Then, a solution of $\mathrm{HSnBu}_{3}(0.05 \mathrm{~mL}, 0.19 \mathrm{mmol}, 1.5$ equiv) in dry, deoxygenated toluene (1.4 mL) was slowly added in $6 \mathrm{~h}$, at $120{ }^{\circ} \mathrm{C}$. The mixture was cooled, and the solvent was evaporated. The residue was dissolved in ethyl ether, and treated with a $10 \%$ aqueous KF solution for $12 \mathrm{~h}$. The organic 
layer was separated, and the aqueous layer was extracted with ethyl ether (x3). The organic phases were dried over $\mathrm{MgSO}_{4}$, filtered, and evaporated. The crude was submitted to chromatography $\left(0.5 \% \rightarrow 1 \% \mathrm{CH}_{2} \mathrm{Cl}_{2}: \mathrm{MeOH}\right)$ to give 7-azabicyclo[2.2.1]heptan-7-yl(2chloropyridin-3-yl)methanone (20) $(15.7 \mathrm{mg}, 52 \%)$ and 7-azabicyclo[2.2.1]heptan-7yl(pyridin-3-yl)methanone (21) $(6.1 \mathrm{mg}, 24 \%)$. 20: white solid; $132-4{ }^{\circ} \mathrm{C}$; IR $(\mathrm{KBr}) \vee 3038$, 2954, 1623, 1581, 1399, $1068 \mathrm{~cm}^{-1} ;{ }^{1} \mathrm{H}$ NMR $\left(400 \mathrm{MHz}, \mathrm{CDCl}_{3}\right) \delta 8.45(\mathrm{dd}, J=4.8,2.0 \mathrm{~Hz}, 1 \mathrm{H}$, ), $7.68(\mathrm{dd}, J=7.5,2.0 \mathrm{~Hz}, 1 \mathrm{H}), 7.30(\mathrm{dd}, J=7.5,4.8 \mathrm{~Hz}, 1 \mathrm{H}), 4.84(\mathrm{t}, J=4.7 \mathrm{~Hz}, 1 \mathrm{H}), 3.70(\mathrm{t}$, $J=4.6 \mathrm{~Hz}, 1 \mathrm{H}), 2.03-1.80(\mathrm{~m}, 4 \mathrm{H}), 1.63-1.46(\mathrm{~m}, 4 \mathrm{H}) ;{ }^{13} \mathrm{C} \mathrm{NMR}\left(100 \mathrm{MHz}, \mathrm{CDCl}_{3}\right) \delta 162.1$ (NCO), 150.3 (C6'), 147.7 (C2'), 137.1 (C4'), 132.9 (C3'), 122.6 (C5'), 58.0 (C1*), 53.6 (C4*), 30.7 (2C), 29.2 (2C) (C2, C3, C5, C6); MS (ES) $\mathrm{m} / \mathrm{z}[\mathrm{M}+1]^{+}$237.1, [M+23] 259.0. Anal. Calcd. for $\mathrm{C}_{12} \mathrm{H}_{13} \mathrm{ClN}_{2} \mathrm{O}$ : C, 60.89; H, 5.54; N, 11.84. Found: $\mathrm{C}, 60.65 ; \mathrm{H}, 5.36 ; \mathrm{N}, 11.71$. 21: white solid; 58-60 ${ }^{\circ} \mathrm{C}$; IR $(\mathrm{KBr}) \vee 3018,2950,1620,1413,1140 \mathrm{~cm}^{-1} ;{ }^{1} \mathrm{H}$ NMR $\left(\mathrm{CDCl}_{3}, 300 \mathrm{MHz}\right)$ $\delta 8.80$ (s, 1H, H2'), 8.69 (d, $J=3.9 \mathrm{~Hz}, 1 \mathrm{H}, \mathrm{H6}$ '), 7.90 (dm, $J=7.8 \mathrm{~Hz}, 1 \mathrm{H}, \mathrm{H} 4$ '), 7.37 (dd, $J=$ 5.0, 7.7 Hz, 1H, H5'), 4.78 (br s, 1H, H1*), 4.11 (br s, 1H, H4*), 2.08-1.70 (m, 4H), 1.61-1.46 (m, 4H); ${ }^{13} \mathrm{C}$ NMR $\left(\mathrm{CDCl}_{3}, 125 \mathrm{MHz}\right) \delta 166.1(\mathrm{NCO}), 151.5$ (C6'), $148.8\left(\mathrm{C2}^{\prime}\right), 135.7\left(\mathrm{C}^{\prime}\right)$, 132.2 (C3'), $123.6\left(\mathrm{C}^{\prime}\right), 59.2\left(\mathrm{C}^{*}\right), 54.2(\mathrm{C} 4 *), 30.8\left(2 \mathrm{xCH}_{2}\right), 28.9\left(2 \mathrm{xCH}_{2}\right)$; MS (ES) $\mathrm{m} / \mathrm{z}$ $[\mathrm{M}+1]^{+}$203.1, $[\mathrm{M}+23]^{+}$225.1. Anal. Calcd. for $\mathrm{C}_{12} \mathrm{H}_{14} \mathrm{~N}_{2} \mathrm{O}: \mathrm{C}, 71.26 ; \mathrm{H}, 6.98 ; \mathrm{N}, 13.85$. Found: C, 70.98; H, 7.10; N, 13.56 .

Synthesis of radical precursor 6. $\boldsymbol{N}$-(Cyclohex-3-enyl)acrylamide $24 .^{21}$ To a solution of 3cyclohex-3-enecarboxylic acid (22) $(240 \mathrm{mg}, 1.91 \mathrm{mmol})$ in dry toluene $(6 \mathrm{~mL}, 0.32 \mathrm{M}), \mathrm{Et}_{3} \mathrm{~N}$ $(0.32 \mathrm{~mL}, 2.29 \mathrm{mmol}, 1.2$ equiv) and DPPA $(0.43 \mathrm{~mL}, 2.00 \mathrm{mmol}, 1.05$ equiv) were added, and the mixture was stirred at $\mathrm{rt}$ for $30 \mathrm{~min}$, and at $80^{\circ} \mathrm{C}$ for $4 \mathrm{~h}$. The mixture was cooled, and acrylic acid ( $0.65 \mathrm{~mL}, 9.53 \mathrm{mmol}, 5.0$ equiv) and $\mathrm{CuCl}(22 \mathrm{mg}, 0.22 \mathrm{mmol}, 0.1$ equiv) were added. The mixture was refluxed for $2 \mathrm{~h}$, and then cooled. An aqueous saturated $\mathrm{NaHCO}_{3}$ solution was added, and extracted with ethyl ether (x3). The organic phase was dried over $\mathrm{Na}_{2} \mathrm{SO}_{4}$, filtered, and evaporated. The crude obtained was submitted to column chromatography (silica gel, $1 \%$ $\mathrm{CH}_{2} \mathrm{Cl}_{2}: \mathrm{MeOH}$ ), to give 1,3-di(cyclohex-3-enyl)urea 23 (28 mg, 14\%), ${ }^{10}$ and compound $24^{21}$ (189 mg, 66\%): white solid; $95-7^{\circ} \mathrm{C}$; IR (KBr) $\vee 3429,3285,3024,2920,1656,1624,1552 \mathrm{~cm}^{-}$ '; ${ }^{1} \mathrm{H}$ NMR $\left(\mathrm{CDCl}_{3}, 400 \mathrm{MHz}\right) \oint 6.24$ (dd, $\left.J=1.6,16.8 \mathrm{~Hz}, 1 \mathrm{H}, \mathrm{H} 3{ }^{\prime}{ }_{\text {cis }}\right), 6.10$ (dd, $J=10.3,17.0$ Hz, 1H, H2'), 5.89 (br s, 1H, NH), 5.68 (dm, $J=9.8 \mathrm{~Hz}, 1 \mathrm{H}, \mathrm{H} 4), 5.63-5.56$ (m, 1H, H3), 5.58 (dd, $J=1.6,10.2 \mathrm{~Hz}, 1 \mathrm{H}, \mathrm{H}^{\prime}{ }_{\text {trans }}$ ), 4.22-4.12 (m, $\left.1 \mathrm{H}, \mathrm{H1}\right), 2.40(\mathrm{dm}, J=17.4 \mathrm{~Hz}, 1 \mathrm{H}, \mathrm{H} 2 \mathrm{~A})$, 2.23-2.01 (m, 2H, 2xH5), 1.96-1.83 (m, 2H, H2 $\left.2_{\mathrm{ax}}, \mathrm{H} 6_{\mathrm{eq}}\right), 1.67-1.55$ (m, 1H, $\left.\mathrm{H} 6_{\mathrm{ax}}\right) ;{ }^{13} \mathrm{C} \mathrm{NMR}$ $\left(\mathrm{CDCl}_{3}, 100 \mathrm{MHz}\right) \delta 165.1(\mathrm{NCO}), 131.5,131.4\left(\mathrm{C} 2{ }^{\prime}\right), 127.2$ (C4), 126.0 (C3'), 124.5 (C3), 44.8, 44.7 (C1), 31.7 (C2), 28.1 C6, 29.6 (C5); MS (ES) $\mathrm{m} / \mathrm{z}[\mathrm{M}+1]^{+}$152.1, [M+23] ${ }^{+}$174.1, $[2 \mathrm{M}+1]^{+} 303.2 ;[2 \mathrm{M}+23]^{+}$325.2. Anal. Calcd. for $\mathrm{C}_{9} \mathrm{H}_{13} \mathrm{NO}$ : C, 71.49; $\mathrm{H}, 8.67 ; \mathrm{N}, 9.26$. Found: C, 71.28; H, 8.61; N, 9.44.

Bromination of $\mathbf{N}$-(cyclohex-3-enyl)acrylamide 24. To a solution of compound 24 (136 $\mathrm{mg}$, $0.90 \mathrm{mmol})$ in dry $\mathrm{CH}_{2} \mathrm{Cl}_{2}(10 \mathrm{~mL}, 0.09 \mathrm{M})$, at $\mathrm{rt}$ and under argon, $\mathrm{Et}_{4} \mathrm{NBr}(1.89 \mathrm{~g}, 8.98 \mathrm{mmol}$, 10 equiv) was added. The mixture was cooled at $-78^{\circ} \mathrm{C}$, and bromine $(0.05 \mathrm{~mL}, 0.99 \mathrm{mmol}, 1.1$ 
equiv) was added. After $1.5 \mathrm{~h}$, the reaction was warmed at $\mathrm{rt}$ and aqueous saturated $\mathrm{Na}_{2} \mathrm{~S}_{2} \mathrm{O}_{5}$ was added. The mixture was then stirred until total decoloration, and extracted with AcOEt (x3). The organic phases were dried over $\mathrm{Na}_{2} \mathrm{SO}_{4}$, filtered, evaporated, and the crude submitted to chromatography $\quad(35 \% \quad$ hexane: AcOEt $), \quad$ giving $N$-(trans-3,cis-4dibromocyclohexyl)acrylamide (25) (63 $\quad \mathrm{mg}, \quad 23 \%)$ and $\quad \boldsymbol{N}$-(cis-3,trans-4dibromocyclohexyl)acrylamide (26) (163 mg, 58\%). 25: white solid; $142-4{ }^{\circ} \mathrm{C}$; IR (KBr) v 3264, 3062, 2951, 1658, 1629, 1544, 1434, $1409 \mathrm{~cm}^{-1} ;{ }^{1} \mathrm{H} \mathrm{NMR}\left(\mathrm{CDCl}_{3}, 400 \mathrm{MHz}\right) \delta 6.31$ (dd, $J=1.4,17.0 \mathrm{~Hz}, 1 \mathrm{H}, \mathrm{H} 3$ ', 6.08 (dd, $J=10.3,17.0 \mathrm{~Hz}, 1 \mathrm{H}, \mathrm{H} 2$ '), 5.67 (dd, $J=1.4,10.3 \mathrm{~Hz}, 1 \mathrm{H}$, H3'), 5.50 (br s, 1H, NH), 4.71-4.57 (m, 2H, H3, H4), 4.51-4.39 (br s, 1H, H1), 2.62 (ddt, J= 3.1, 12.5, 15.6 Hz, 1H, H5 ax), 2.38 (ddd, $J=3.1,11.3,14.4 \mathrm{~Hz}, 1 \mathrm{H}, \mathrm{H} 2_{\mathrm{ax}}$ ), 2.26 (dm, J=14.1 Hz, 1H, $\left.\mathrm{H} 2_{\mathrm{eq}}\right), 2.09-1.92\left(\mathrm{~m}, 2 \mathrm{H}, \mathrm{H} 5_{\mathrm{eq}}, \mathrm{H} 6_{\mathrm{eq}}\right), 1.78\left(\mathrm{qd}, J=3.5,12.4 \mathrm{~Hz}, 1 \mathrm{H}, \mathrm{H} 6_{\mathrm{ax}}\right) ;{ }^{13} \mathrm{C} \mathrm{NMR}\left(\mathrm{CDCl}_{3}\right.$, $100 \mathrm{MHz}) \oint 164.9$ (C1'), 130.8 (C2’), 127.1 (C3'), 52.4 (C4), 51.4 (C3), 43.9 (C1), 34.8 (C2), 28.3 (C5), 27.3 (C6); MS (ES) m/z [M+1] 309.9/311.9/313.9, [M+23] 331.9/333.9/335.9. Anal. Calcd. for $\mathrm{C}_{9} \mathrm{H}_{13} \mathrm{Br}_{2} \mathrm{NO}$ : C, 34.76; H, 4.21; N, 4.50. Found: C, 34.95; H, 4.33; N, 4.71. (26): white solid; $119-121{ }^{\circ} \mathrm{C}$; IR $(\mathrm{KBr}) \vee 3422,3260,3071,2947,1655,1624,1552,1410 \mathrm{~cm}^{-1}$; ${ }^{1} \mathrm{H}$ NMR $\left(\mathrm{CDCl}_{3}, 400 \mathrm{MHz}\right) \oint 6.28(\mathrm{dd}, J=1.3,17.0 \mathrm{~Hz}, 1 \mathrm{H}, \mathrm{H} 3$ '), 6.06 (dd, J=10.2, $17.0 \mathrm{~Hz}, 1 \mathrm{H}$, H2'), 5.94 (br s, 1H, NH), 5.67 (dd, $J=1.4,10.3 \mathrm{~Hz}, 1 \mathrm{H}, \mathrm{H} 3$ '), 4.24 (td, $J=4.2,9.1 \mathrm{~Hz}, 1 \mathrm{H}, \mathrm{H} 3$ ), 4.20-4.30 (m, 2H, H1, H4), 2.80 (ddt, $J=2.0,4.2,13.7 \mathrm{~Hz}, 1 \mathrm{H}, \mathrm{H} 2_{\mathrm{ec}}$ ), 2.52 (ddt, J= 3.9, 5.7, 14.3 $\left.\mathrm{Hz}, 1 \mathrm{H}, \mathrm{H} 5_{\mathrm{eq}}\right), 2.09$ (dm, J= 13.3 Hz, 1H, H6 $6_{\mathrm{eq}}$ ), 2.04-1.93 (m, 1H, H5 ax), 1.91 (dt, J= 9.7, 13.7 $\left.\mathrm{Hz}, 1 \mathrm{H}, \mathrm{H} 2_{\mathrm{ax}}\right), 1.53-1.41\left(\mathrm{~m}, 1 \mathrm{H}, \mathrm{H} 6_{\mathrm{ax}}\right) ;{ }^{13} \mathrm{C} \mathrm{NMR}\left(\mathrm{CDCl}_{3}, 100 \mathrm{MHz}\right) . \oint 164.8\left(\mathrm{C} 1{ }^{\prime}\right), 130.8$ (C2'), 127.2 (C3'), 54.8 (C4), 52.1 (C3), 46.3 (C1), 41.0 (C2), 33.1 (C5), 30.9 (C6); MS (ES) $\mathrm{m} / \mathrm{z}[\mathrm{M}+1]^{+} 309.9 / 311.9 / 313.9,[\mathrm{M}+23]^{+}$331.9/333.9/335.9. Anal. Calcd. for $\mathrm{C}_{9} \mathrm{H}_{13} \mathrm{Br}_{2} \mathrm{NO}: \mathrm{C}$, 34.76; H, 4.21; N, 4.50. Found: C, 34.80; H, 4.45; N, 4.60 .

1-(2-Bromo-7-azabicyclo[2.2.1]heptan-7-yl)prop-2-en-1-one (6). To a solution of compound 26 (50 mg, $0.16 \mathrm{mmol})$ in dry DMF $(2 \mathrm{~mL}, 0.09 \mathrm{M}), \mathrm{NaH}(8 \mathrm{mg}, 0.20 \mathrm{mmol}, 1.2$ equiv, 60\% in oil) was added at $0{ }^{\circ} \mathrm{C}$. The resulting mixture was stirred at $\mathrm{rt}$ for $14 \mathrm{~h}$. The addition of $\mathrm{NaH}(4$ $\mathrm{mg}, 0.09 \mathrm{mmol}, 0.6$ equiv) was repeated, and the reaction stirred for $7 \mathrm{~h}$ more; then, water was added, and extracted with ethyl ether $(\mathrm{x} 4)$. The organic phase was washed with brine, dried over $\mathrm{MgSO}_{4}$, filtered and evaporated. The residue was submitted to chromatography $\left(0.5 \% \mathrm{CH}_{2} \mathrm{Cl}_{2}\right.$ : $\mathrm{MeOH})$ to give precursor 6 (19 mg, 52\%): oil; IR (film) $\vee 2951,1651,1614,1437 \mathrm{~cm}^{-1} ;{ }^{1} \mathrm{H}$ NMR $\left(\mathrm{CDCl}_{3}, 500 \mathrm{MHz}\right) \delta .6 .46(\mathrm{dd}, J=9.8,16.9 \mathrm{~Hz}, 1 \mathrm{H}, \mathrm{H} 2$ '), 6.39 (br d, $J=16.6 \mathrm{~Hz}, 1 \mathrm{H}$, H3 ' ${ }_{\text {cis }}$ ), 5.72 (br d, J= $10.0 \mathrm{~Hz}, 1 \mathrm{H}, \mathrm{H} 3{ }_{\text {trans }}$ ), 4.90 (br s, H1, minor invertomer), 4.82 (s, H4, major invertomer), 4.44 (d, $J=4.6 \mathrm{~Hz}, \mathrm{H1}$, major invertomer), 4.40 (br s, H4, minor invertomer), 4.14-4.07 (m, H2, major invertomer), 4.04 (br s, H2, minor invertomer), 2.38-2.19 (m, 2H, $2 \mathrm{xH} 3$ ), 1.98-1.88 (m, 1H, H6 $6_{\text {exo }}$ ), 1.85-1.66 (m, 1H, H5 exo), 1.61-1.33 (m, 2H, H5 endo, $\mathrm{H}_{\text {endo }}$ ); ${ }^{13} \mathrm{C} \mathrm{NMR}\left(\mathrm{CDCl}_{3}, 125 \mathrm{MHz}\right) \oint 163.8$ (NCO, C1'), 128.6 (C2'), 127.9 (C3'), 64.3, 61.5 (C1), 56.3, 53.6 (C4), 50.2, 47.8 (C2), 44.6, 42.7 (C3), 29.8, 29.1 (C6), 27.5, 26.8 (C5); MS (ES) m/z $[\mathrm{M}+1]^{+}$230.1/232.1, $[\mathrm{M}+23]^{+}$252.1/254.1, $[2 \mathrm{M}+23]^{+}$481.0/483.0/485.0. Anal. Calcd. for $\mathrm{C}_{9} \mathrm{H}_{12}$ BrNO: C, 46.98; H, 5.26; N, 6.09. Found: C, 46.79; H, 5.31; N, 6.32. 
$\mathbf{N}$-(Cyclohex-3-enyl)acetamide (27). To a solution of cyclohex-3-en carboxylic acid 22 (156 $\mathrm{mg}, 1.23 \mathrm{mmol})$ in dry toluene $(6 \mathrm{~mL}, 0.21 \mathrm{M})$, dry Et $\mathrm{H}_{3} \mathrm{~N}(0.21 \mathrm{~mL}, 1.51 \mathrm{mmol}, 1.22$ equiv) and DPPA $(0.29 \mathrm{~mL}, 1.30 \mathrm{mmol}, 1.06 \mathrm{mmol})$ were added, and the mixture was stirred at $\mathrm{rt}$ for 30 min. Then, the reaction was refluxed for $5 \mathrm{~h}$, the flask was cooled, and acetic acid $(0.35 \mathrm{~mL}, 6.17$ mmol, 5 equiv) and $\mathrm{CuCl}(9.3 \mathrm{mg}, 0.09 \mathrm{mmol}, 0.08$ equiv) were added. The mixture was refluxed again for $2 \mathrm{~h}$. Then, the flask was cooled, aqueous saturated $\mathrm{NaHCO}_{3}$ was added, and the solution was extracted with ethyl ether (x4). The organic phase was dried over $\mathrm{Na}_{2} \mathrm{SO}_{4}$, filtered, and evaporated. The residue was submitted to chromatography $(30 \% \rightarrow 50 \%$ hexane: AcOEt) giving 1,3-di(cyclohex-3-enyl)urea $23^{10}$ (27.5 mg, 20\%), and compound $27^{22}$ (111 mg, 65\%): white solid; $75-7^{\circ} \mathrm{C}$; IR (KBr) v 3298, 3079, 3032, 2928, 2840, 1645, 1554, 1311, 655 $\mathrm{cm}^{-1}$; ${ }^{1} \mathrm{H}$ NMR $\left(\mathrm{CDCl}_{3}, 400 \mathrm{MHz}\right) \delta$ 5.72-5.66 (m, 1H, H4), 5.64-5.54 (m, 2H, H3, NH), 4.164.06 (m, 1H, H1), 2.38 (dm, J=17.3 Hz, 1H, H2ax), 2.23-2.04 (m, 2H, 2xH5), $1.98\left(\mathrm{~s}, 3 \mathrm{H}, \mathrm{CH}_{3}\right)$, 1.92-1.81 (m, 2H, H2 $\left.2_{\text {eq }}, \mathrm{H} 6_{\mathrm{ax}}\right), 1.63-1.52\left(\mathrm{~m}, 1 \mathrm{H}, \mathrm{H} 6_{\mathrm{eq}}\right) ;{ }^{13} \mathrm{C} \mathrm{NMR}\left(\mathrm{CDCl}_{3}, 100 \mathrm{MHz}\right) \delta 169.6$ (NHCO), 127.2 (C4), 124.5 (C3), 44.6 (C1), 31.8 (C2), 28.1 (C6), $23.7\left(\mathrm{CH}_{3}\right), 23.6$ (C5); MS (ES) $\mathrm{m} / \mathrm{z}[\mathrm{M}+1]^{+}$140.1, $[\mathrm{M}+23]^{+}$162.1, $[2 \mathrm{M}+1]^{+}$279.3, $[2 \mathrm{M}+23]^{+}$301.2. Anal. Calcd. for $\mathrm{C}_{8} \mathrm{H}_{13} \mathrm{NO} \cdot 1 / 3 \mathrm{H}_{2} \mathrm{O}: \mathrm{C}, 66.17 ; \mathrm{H}, 9.49 ; \mathrm{N}, 9.65$. Found: C, 65.86; H, 9.22; N, 9.92.

Bromination of $\mathbf{N}$-(cyclohex-3-enyl)acetamide (27). To a solution of compound 27 (220 mg, $1.58 \mathrm{mmol})$ in dry $\mathrm{CH}_{2} \mathrm{Cl}_{2}(19 \mathrm{~mL}, 0.08 \mathrm{M}), \mathrm{Et}_{4} \mathrm{NBr}(3.34 \mathrm{~g}, 0.016 \mathrm{~mol}, 10$ equiv) was added, and the mixture was stirred under argon for some minutes. After cooling at $-78{ }^{\circ} \mathrm{C}, \mathrm{Br}_{2}(0.16 \mathrm{~mL}$, $3.1 \mathrm{mmol}, 2.0$ equiv) was added, and the mixture was stirred for $2 \mathrm{~h}$. The reaction was warmed at $\mathrm{rt}$, and then an aqueous saturated $\mathrm{Na}_{2} \mathrm{~S}_{2} \mathrm{O}_{5}$ solution was added until the colour was quenched. The mass was extracted with AcOEt (x3), and the organic phase was dried over $\mathrm{Na}_{2} \mathrm{SO}_{4}$, filtered, and evaporated. The crude was submitted to chromatography (75\% hexane: AcOEt) affording $N$ (trans-3,cis-4-dibromocyclohexyl)acetamide 28 (108 $\mathrm{mg}, 23 \%)$, and $N$-(cis-3,trans-4dibromocyclohexyl]acetamide 29 (306 mg, 64.5\%). 28: white solid; $118-120{ }^{\circ} \mathrm{C}$; IR (KBr) v 3257, 2953, 1635, 1566, 1310, $545 \mathrm{~cm}^{-1} ;{ }^{1} \mathrm{H}$ NMR $\left(\mathrm{CDCl}_{3}, 400 \mathrm{MHz}\right) \delta 5.55$ (br s, $\left.1 \mathrm{H}, \mathrm{NH}\right)$, $4.66-4.58(\mathrm{~m}, 2 \mathrm{H}, \mathrm{H} 3, \mathrm{H} 4), 4.40-4.28\left(\mathrm{~m}, \mathrm{~W}_{\text {full }} \approx 45 \mathrm{~Hz}, 1 \mathrm{H}, \mathrm{H} 1\right), 2.59$ (ddt, $J=3.5,12.6,15.6$ $\mathrm{Hz}, 1 \mathrm{H}, \mathrm{H} 5_{\mathrm{eq}}$ ), 2.33 (ddd, J=3.2, 11.2, $14.4 \mathrm{~Hz}, 1 \mathrm{H}, \mathrm{H} 2_{\mathrm{ax}}$ ), 2.20 (dm, J=14.2 Hz, 1H, H2 ec), 1.99 $\left(\mathrm{s}, 3 \mathrm{H}, \mathrm{CH}_{3}\right), 2.05-1.88\left(\mathrm{~m}, 2 \mathrm{H}, \mathrm{H} 5_{\mathrm{ax}}, \mathrm{H} 6_{\mathrm{eq}}\right), 1.79-1.66\left(\mathrm{~m}, 1 \mathrm{H}, \mathrm{H} 6_{\mathrm{ax}}\right) ;{ }^{13} \mathrm{C} \mathrm{NMR}\left(\mathrm{CDCl}_{3}, 100\right.$ $\mathrm{MHz}) \delta 169.5$ (NHCO), $52.4(\mathrm{C} 3 *), 51.5\left(\mathrm{C}^{*}\right), 43.8$ (C1), 34.8 (C2), 28.3 (C5), 27.4 (C6), 23.7 $\left(\mathrm{CH}_{3}\right)$; MS (ES) $\mathrm{m} / \mathrm{z}[\mathrm{M}-\mathrm{Br}]^{+}$218.1/220.1; [M+1] ${ }^{+}$298.0/300.0/302.0, $[\mathrm{M}+23]^{+}$320/322/324. Anal. Calcd. for $\mathrm{C}_{8} \mathrm{H}_{13} \mathrm{Br}_{2} \mathrm{NO}$ : C, 32.14; H, 4.38; N, 4.68; O, 5.35. Found: C, 32.32; H, 4.14; N, 4.74. 29: white solid; $106-8{ }^{\circ} \mathrm{C}$; IR $(\mathrm{KBr}) \vee 3255,2951,1636,1579,1373,1168,688 \mathrm{~cm}^{-1} ;{ }^{1} \mathrm{H}$ NMR $\left(\mathrm{CDCl}_{3}, 400 \mathrm{MHz}\right) \delta 5.73($ br s, $1 \mathrm{H}, \mathrm{NH}), 4.19$ (td, $\left.J=4.2,9.6 \mathrm{~Hz}, 1 \mathrm{H}, \mathrm{H} 3\right), 4.11$ (td, $J=$ 4.0, $9.7 \mathrm{~Hz}, 1 \mathrm{H}, \mathrm{H} 4), 4.04-3.93\left(\mathrm{~m}, \mathrm{~W}_{\text {full }} \approx 43 \mathrm{~Hz}, 1 \mathrm{H}, \mathrm{H1}\right), 2.76(\mathrm{dtd}, J=2.1,4.2,13.6 \mathrm{~Hz}, 1 \mathrm{H}$, $\left.\mathrm{H} 2_{\mathrm{eq}}\right), 2.50\left(\mathrm{dm}, J=14.3 \mathrm{~Hz}, 1 \mathrm{H}, \mathrm{H} 5_{\mathrm{ec}}\right), 2.09-1.99\left(\mathrm{~m}, 1 \mathrm{H}, \mathrm{H} 6_{\mathrm{eq}}\right), 1.99-1.96\left(\mathrm{~m}, 1 \mathrm{H}, \mathrm{H} 5_{\mathrm{ax}}\right), 1.97$ $\left(\mathrm{s}, 3 \mathrm{H}, \mathrm{CH}_{3}\right), 1.85\left(\mathrm{dt}, J=9.8,13.5 \mathrm{~Hz}, 1 \mathrm{H}, \mathrm{H} 2_{\mathrm{ax}}\right), 1.40\left(\mathrm{~m}, 1 \mathrm{H}, \mathrm{H} 6_{\mathrm{ax}}\right) ;{ }^{13} \mathrm{C} \mathrm{NMR}\left(\mathrm{CDCl}_{3}, 100\right.$ $\mathrm{MHz}) \delta 169.5$ (NHCO), 55.1 (C4), 53.5 (C3), 46.8 (C1), 42.0 (C2), 34.0 (C5), 31.4 (C6), 23.4 $\left(\mathrm{CH}_{3}\right) ; \mathrm{MS}$ (ES) $\mathrm{m} / \mathrm{z}[\mathrm{M}+1]^{+}$298.0/300.0/302.0, [M+23] $]^{+}$320.0/322.0/324.0. Anal. Calcd. $\mathrm{C}_{8} \mathrm{H}_{13} \mathrm{Br}_{2} \mathrm{NO}$ : C, 32.14; H, 4.38; N, 4.68. Experimental: $\mathrm{C}, 31.89 ; \mathrm{H}, 4.31 ; \mathrm{N}, 4.69$. 
1-(2-Bromo-7-azabicyclo[2.2.1]heptan-7-yl)ethanone (30). To a solution of compound 29 (109 $\mathrm{mg}, 0.36 \mathrm{mmol})$ in dry DMF, under argon and at $0{ }^{\circ} \mathrm{C}$, NaH (16 mg, $0.4 \mathrm{mmol}, 1.1 \mathrm{equiv}, 60 \%$ dispersion in oil) was added. After $40 \mathrm{~min}$, the mixture was stirred at rt for $19 \mathrm{~h}$; then, $\mathrm{NaH}(8.0$ $\mathrm{mg}, 0.2 \mathrm{mmol}, 0.55$ equiv) was added again, and the stirring was maintained for $24 \mathrm{~h}$ at $\mathrm{rt}$. The mixture was cooled, water was added, extracted with ethyl ether (x4), and the organic phase was washed with brine, dried over $\mathrm{MgSO}_{4}$, filtered, and evaporated. The crude was submited to chromatography $\left(1 \% \rightarrow 2 \% \mathrm{CH}_{2} \mathrm{Cl}_{2}\right.$ : $\left.\mathrm{MeOH}\right)$ giving compound 30 (34.3 mg, 43\%), identical in all its analytical and spectroscopic data for the same compound obtained by a different route in our laboratory. ${ }^{9}$

$\mathbf{N}$-(Cyclohex-3-enyl)-4-methylbenzenesulfonamide (32). To a solution of carbamate $\mathbf{3 1}{ }^{9}$ (726 $\mathrm{mg}, 3.68 \mathrm{mmol})$ in dry $\mathrm{CH}_{2} \mathrm{Cl}_{2}(52 \mathrm{~mL}, 0.07 \mathrm{M})$ under argon and at $\mathrm{rt}$, trifluoroacetic acid (5.28 mL, $71.07 \mathrm{mmol}, 19.3$ equiv) was added, and the mixture was stirred for $2.5 \mathrm{~h}$. The solvent was evaporated to give pure primary amine ${ }^{[15]}$ as its trifluoroacetate salt [white solid; $121-3{ }^{\circ} \mathrm{C}$; IR $(\mathrm{KBr}) \vee 3430,3038,1699,1178,724 \mathrm{~cm}^{-1} ;{ }^{1} \mathrm{H}$ NMR $\left(\mathrm{CDCl}_{3}, 400 \mathrm{MHz}\right) \delta 5.73-5-65(\mathrm{~m}, 1 \mathrm{H}$, H4), 5.61-5.53 (m, 1H, H3), 3.35-3.24 (m, 1H, H1), 2.38 (dm, J=17.0 Hz, 1H, H2A), 2.19-2.11 (m, 2H, 2xH5), 2.09-1.91 (m, 2H, H2B, H6A), 1.68-1.55 (m, 1H, H6B); 5.68-5.58 (m, 1H, H4), 5.53-5.45 (m, 1H, H3), 4.87 (d, J=8.1 Hz, 1H, NH), 3.49-3.37 (m, 1H, H1), $2.43\left(\mathrm{~s}, 3 \mathrm{H}, \mathrm{CH}_{3}\right)$, $2.19(\mathrm{dm}, J=17.4 \mathrm{~Hz}, 1 \mathrm{H}, \mathrm{H} 2 \mathrm{~A}), 2.13-1.95$ (m, 2H, 2xH5), 1.84 (dm, J=17.4 Hz, 1H, H2B), $1.74(\mathrm{dm}, J=12.8 \mathrm{~Hz}, 1 \mathrm{H}, \mathrm{H} 6 \mathrm{~A}), 1.60-1.47$ (m, $1 \mathrm{H}, \mathrm{H} 6 \mathrm{~B}) ;{ }^{13} \mathrm{C} \mathrm{NMR}\left(\mathrm{CDCl}_{3}, 100 \mathrm{MHz}\right) \delta 128.1$ (C4), 123.7 (C3), 48.3 (C1), 30.4 (C2), 27.6 (C6), 24.4 (C5). Anal. Calcd. for $\mathrm{C}_{6} \mathrm{H}_{11} \mathrm{~N} \cdot 5 \mathrm{CF}_{3} \mathrm{COOH}$ : C, 28.80; H, 2.42; N, 2.10. Found: C, 28.17; H, 2.95; N, 2.97]. The resulting crude was dissolved in dry $\mathrm{CH}_{2} \mathrm{Cl}_{2}(20 \mathrm{~mL}, 0.18 \mathrm{M})$ under argon and at $0{ }^{\circ} \mathrm{C}$. Then, $\mathrm{Et}_{3} \mathrm{~N}$ (1.3 mL, $9.33 \mathrm{mmol}, 2.5$ equiv) and $p$ - $\mathrm{TsCl}$ ( $878 \mathrm{mg}, 4.60 \mathrm{mmol}, 1.25$ equiv) were added. The mixture was stirred at $\mathrm{rt}$ for $48 \mathrm{~h}$. An aqueous $\mathrm{H}_{2} \mathrm{SO}_{4}(9.39 \mathrm{~mL}, 2 \mathrm{M})$ solution was then added, followed by an aqueous saturated $\mathrm{NaHCO}_{3}$ solution (until $\mathrm{pH}$ 7), and the mixture was extracted with $\mathrm{CH}_{2} \mathrm{Cl}_{2}$. The organic phase was dried over $\mathrm{MgSO}_{4}$, filtered, and evaporated to give a crude that was submitted to chromatography (20\% hexane: AcOEt) giving sulfonamide $32^{23}$ (916 mg, 99\%): oil; IR (KBr) v 3273, 3022, 2918, 1438, 1323, $1159 \mathrm{~cm}^{-1}$; ${ }^{1} \mathrm{H}$ NMR $\left(\mathrm{CDCl}_{3}\right.$, $400 \mathrm{MHz}) \delta 7.78$ (d, J=8.5 Hz, 2H, H2', H6'), 7.30 (d, J=8.6 Hz, 2H, H3', H5'), 5.68-5.58 (m, 1H, H4), 5.53-5.45 (m, 1H, H3), 4.87 (d, J=8.1 Hz, 1H, NH), 3.49-3.37 (m, 1H, H1), 2.43 (s, $\left.3 \mathrm{H}, \mathrm{CH}_{3}\right), 2.19$ (dm, $\left.J=17.4 \mathrm{~Hz}, 1 \mathrm{H}, \mathrm{H} 2 \mathrm{ax}\right), 2.13-1.95$ (m, 2H, 2xH5), 1.84 (dm, J=17.4 Hz, 1H, $\left.\mathrm{H} 2_{\mathrm{eq}}\right), 1.74\left(\mathrm{dm}, J=12.8 \mathrm{~Hz}, 1 \mathrm{H}, \mathrm{H} 6_{\mathrm{eq}}\right), 1.60-1.47\left(\mathrm{~m}, 1 \mathrm{H}, \mathrm{H} 6_{\mathrm{ax}}\right) ;{ }^{13} \mathrm{C} \mathrm{NMR}\left(\mathrm{CDCl}_{3}, 100\right.$ MHz) $\delta 143.4$ (C4'), 138.4 (C1'), 129.8 (2xCH, C3', C5'), 127.2 (C4), 127.1 (2xCH, C2', C6'), 124.0 (C3), 49.0 (C1), 32.5 (C2), 28.9 (C6), 23.5 (C5), $21.7\left(\mathrm{CH}_{3}\right)$; MS (ES) m/z [M+1] 252.1, $[\mathrm{M}+23]^{+}$274.0, $[2 \mathrm{M}+23]^{+}$525.3. Calcd. for $\mathrm{C}_{13} \mathrm{H}_{17} \mathrm{NO}_{2} \mathrm{~S}: \mathrm{C}, 62.12 ; \mathrm{H}, 6.82 ; \mathrm{N}, 5.57, \mathrm{~S}, 12.76$. Found: C, 61.98; H, 6.99; N, 5.33; S, 12.52 .

Bromination of $\mathbf{N}$-(cyclohex-3-enyl)-4-methylbenzenesulfonamide (32). To a solution of compound 32 (475 mg, $1.89 \mathrm{mmol}$ ) and $\mathrm{Et}_{4} \mathrm{NBr}\left(3.974 \mathrm{~g}, 18.89 \mathrm{mmol}, 10\right.$ equiv) in dry $\mathrm{CH}_{2} \mathrm{Cl}_{2}$ $(22 \mathrm{~mL}, 0.086 \mathrm{M})$ at $-78{ }^{\circ} \mathrm{C}$ and under argon, bromine $(0.11 \mathrm{~mL}, 2.08 \mathrm{mmol}, 1.1$ equiv) was added. The mixture was stirred for $4 \mathrm{~h}$, and then warmed at rt. An aqueous saturated $\mathrm{Na}_{2} \mathrm{~S}_{2} \mathrm{O}_{5}$ 
solution was then added until total decolouration. The solution was extracted with AcOEt ( $\mathrm{x} 4)$, the organic phase was dried over $\mathrm{Na}_{2} \mathrm{SO}_{4}$, filtered, and evaporated. The crude was submitted to chromatography $(10 \%$ hexane: AcOEt) giving $\mathrm{N}$-(cis-3,trans-4-dibromocyclohexyl)-4methylbenzenesulfonamide (33) (546 mg, 70\%) and $\mathrm{N}$-(trans-3,cis-4-dibromocyclohexyl)-4methylbenzenesulfonamide (34) (154 mg, 20\%). 33: White solid; 137-9 ${ }^{\circ} \mathrm{C}$; IR (KBr) $\vee 3436$, 3226, 3057, 2952, 1450, 1330, $1154 \mathrm{~cm}^{-1}$; ${ }^{1} \mathrm{H} \mathrm{NMR}\left(\mathrm{CDCl}_{3}, 400 \mathrm{MHz}\right) \delta 7.76(\mathrm{~d}, J=8.2 \mathrm{~Hz}, 2 \mathrm{H}$, H2', H6'), 7.32 (d, J= 8.1 Hz, 2H, H3', H5'), 5.41 (d, J= 7.9 Hz, 1H, NH), 4.05-3.92 (m, 2H, H3, H4), 3.30-3.18 (br s, 1H, H1), 2.56 (dm, $J=13.7 \mathrm{~Hz}, 1 \mathrm{H}, \mathrm{H} 2_{\text {eq }}$ ), 2.44 (s, $3 \mathrm{H}, \mathrm{CH}_{3}$ ), 2.45-2.36 (m, $\left.1 \mathrm{H}, \mathrm{H} 5_{\mathrm{eq}}\right), 1.92-1.74$ (m, 3H, H2 $\left.2_{\mathrm{ax}}, \mathrm{H} 5_{\mathrm{ax}}, \mathrm{H} 6_{\mathrm{eq}}\right), 1.44-1.30$ (m, $\left.1 \mathrm{H}, \mathrm{H6} 6_{\mathrm{ax}}\right) ;{ }^{13} \mathrm{C} \mathrm{NMR}\left(\mathrm{CDCl}_{3}\right.$, $100 \mathrm{MHz}$ ) $\delta 143.9$ (C4'), 137.7 (C1'), 130.0 (2xCH, C3', C5'), 127.0 (2xCH, C2', C6'), 54.6, 52.9 (C3, C4), 50.8 (C1), 42.6 (br, C2), 33.8 (br, C5), 32.4 (C6), $21.7\left(\mathrm{CH}_{3}\right)$; MS (ES) m/z $[\mathrm{M}+1]^{+}$410.0/412.0/414.0, [M+23] $]^{+}$432.0/434.0/436.0. Anal. Calcd. for $\mathrm{C}_{13} \mathrm{H}_{17} \mathrm{Br}_{2} \mathrm{NO}_{2} \mathrm{~S} \mathrm{C}$, 37.98; H, 4.17; N, 3.41; S, 7.81. Found: C, 38.20; H, 4.35; N, 3.27; S, 7.76. 34: White solid; 104$6{ }^{\circ} \mathrm{C}$; IR $(\mathrm{KBr}) \vee 3436,3250,3060,2945,1442,1330,1170 \mathrm{~cm}^{-1} ;{ }^{1} \mathrm{H}$ NMR $\left(\mathrm{CDCl}_{3}, 400 \mathrm{MHz}\right)$ $\delta 7.79$ (d, $J=8.3 \mathrm{~Hz}, 2 \mathrm{H}, \mathrm{H} 2^{\prime}, \mathrm{H} 6$ ') 7.33 (d, $J=8.4 \mathrm{~Hz}, 2 \mathrm{H}, \mathrm{H} 3$ ', H5'), 4.53-4.43 (m, 3H, NH, $\mathrm{H} 3, \mathrm{H} 4), 3.77-3.66$ (m, $1 \mathrm{H}, \mathrm{H1}$ ), 2.52-2.42 (m, 1H, H5 ax), 2.45 (s, 2H, $\mathrm{CH}_{3}$ ), 2.28 (ddd, J= 3.1, 11.1, $\left.14.4 \mathrm{~Hz}, 1 \mathrm{H}, \mathrm{H}, \mathrm{H} 2_{\mathrm{ax}}\right), 2.02-1.92\left(\mathrm{~m}, 2 \mathrm{H}, \mathrm{H}, \mathrm{H} 2_{\mathrm{eq}}, \mathrm{H} 5_{\mathrm{eq}}\right), 1.80-1.72(\mathrm{~m}, 2 \mathrm{H}, 2 \mathrm{xH} 6) ;{ }^{13} \mathrm{C}$ NMR ( $\left.\mathrm{CDCl}_{3}, 100 \mathrm{MHz}\right) \delta 143.8\left(\mathrm{C}^{\prime}\right), 138.1\left(\mathrm{Cl}^{\prime}\right), 130.0\left(2 \mathrm{xCH}, \mathrm{C} 3^{\prime}, \mathrm{C}^{\prime}\right), 127.2(2 \mathrm{xCH}$, C2', C6'), 51.5, 51.4 (C3, C4), 48.2 (C1), 35.8 (C2), 28.5 (C6), 28.4 (C5), $21.8\left(\mathrm{CH}_{3}\right)$; MS (ES) $\mathrm{m} / \mathrm{z}[\mathrm{M}+23]^{+} 432.0 / 434.0 / 436.0$. Anal. Calcd. for $\mathrm{C}_{13} \mathrm{H}_{17} \mathrm{Br}_{2} \mathrm{NO}_{2} \mathrm{~S} \mathrm{C}, 37.98 ; \mathrm{H}, 4.17 ; \mathrm{N}, 3.41$. Found: C, 38.11; H, 4.06; N, 3.24.

2-Bromo-7-tosyl-7-azabicyclo[2.2.1]heptane (7). To a solution of compound 33 (146 mg, 0.356 $\mathrm{mmol})$ in dry DMF $(3.4 \mathrm{~mL}, 0.097 \mathrm{M}), \mathrm{NaH}(17 \mathrm{mg}, 0.42 \mathrm{mmol}, 1.2$ equiv, $60 \%$ in oil) was added at $0{ }^{\circ} \mathrm{C}$. After stirring for $1 \mathrm{~h}$, the mixtured was warmed at $\mathrm{rt}$ for $17 \mathrm{~h}$. Then, more $\mathrm{NaH}$ (7.3 mg, $0.182 \mathrm{mmol}, 0.51$ equiv) was added, and the mixture was reacted for $6 \mathrm{~h}$. Then, water was added, and the mixture extracted with ethyl ether $(\mathrm{x} 4)$. The organic phases were washed with brine, dried over $\mathrm{MgSO}_{4}$, filtered, and evaporated. The crude was submitted to chromatography (hexane: AcOEt 15\%) to give recovered compound $33(20.8 \mathrm{mg})$, and product $7^{18}$ \{81.6 mg [69\% (81\%)]\}: white solid; 107-9 ${ }^{\circ} \mathrm{C}$; IR (KBr) $\vee 3049,2958,1595,1335,1155$, 1092, $1058 \mathrm{~cm}^{-1}$; ${ }^{1} \mathrm{H}$ NMR $\left(\mathrm{CDCl}_{3}, 400 \mathrm{MHz}\right) \delta 7.89$ (d, J= 8.2 Hz, 2H, H2', H6'), 7.31 (d, J= $7.9 \mathrm{~Hz}, 2 \mathrm{H}, \mathrm{H} 3$ ', H5'), 4.28 (t, J= 4.7 Hz, 1H, H4), 4.24 (d, J=5.1 Hz, 1H, H1), 3.93 (dd, J= 3.1, $7.5 \mathrm{~Hz}, 1 \mathrm{H}, \mathrm{H} 2), 2.43$ (s, $3 \mathrm{H}, \mathrm{CH}_{3}$ ), 2.27 (dm, $J=13.7 \mathrm{~Hz}, 1 \mathrm{H}, \mathrm{H} 3_{\text {exo }}$ ), 2.16 (dd, J= 7.5, $13.7 \mathrm{~Hz}$, $1 \mathrm{H}, \mathrm{H} 3_{\text {endo }}$ ), 2.13-2.04 (m, 1H, H6 $6_{\text {exo }}$ ), 2.03-1.93 (m, 1H, H5 exo), 1.53-1.45 (m, 1H, H6 $6_{\text {endo }}$ ), 1.43$1.15\left(\mathrm{~m}, 1 \mathrm{H}, \mathrm{H} 5_{\text {endo }}\right) ;{ }^{13} \mathrm{C} \mathrm{NMR}\left(\mathrm{CDCl}_{3}, 100 \mathrm{MHz}\right) \delta 143.8\left(\mathrm{C} 4\right.$ '), $137.4\left(\mathrm{Cl}^{\prime}\right), 129.6(2 \mathrm{xCH}$, C3', C5'), 127.9 (2xC, C2', C6'), 67.2 (C1), 59.4 (C4), 48.4 (C2), 44.5 (C3), 29.0 (C5), 28.2 (C6), $21.8\left(\mathrm{CH}_{3}\right) ; \mathrm{MS}$ (ES) $\mathrm{m} / \mathrm{z}[\mathrm{M}+1]^{+} 330.0 / 332.0,[\mathrm{M}+23]^{+} 350.0 / 352.0,[2 \mathrm{M}+23]^{+}$ 681.0/683.2/685.0. Anal. Calcd. for $\mathrm{C}_{13} \mathrm{H}_{16} \mathrm{BrNO}_{2} \mathrm{~S}$ : C, 47.28; H, 4.88; N, 4.24; S, 9.71. Found: C, 47.31; H, 4.93; N, 4.18; S, 9.60 . 


\section{Acknowledgements}

JMC thanks MEC (Spain) for a grant (SAF2006-08764-C02-01), Comunidad de Madrid (S/SAL0275-2006), Instituto de Salud Carlos III [RED RENEVAS (RD06/0026/1002)], and CSIC (2008CR0004).

\section{References}

1. For some selected reviews on the chemistry and biology of epibatidine, and epibatidine analogues, see: (a) Daly, J. W. J. Med. Chem. 2003, 46, 445. (b) Romanelli, M. N.; Gualteri, F. Med. Res. Rev. 2003, 23, 393. (c) Broka, C. A. Med. Chem. Res. 1994, 4, 449.

2. Spande, T. F.; Garraffo, H. M.; Edwards, M. W.; Yeh, H. J. C.; Pannell, L.; Daly, J. W. J. Am. Chem. Soc. 1992, 114, 3475.

3. Holladay, M. W.; Dart, M. J.; Lynch, J. K. J. Med. Chem. 1997, 40, 4169.

4. Rao, T. S .; Correa, L. D.; Reid, R. T.; Lloyd, G. K. Neuropharmacology 1996, 35, 393.

5. (a) Chen, Z.; Trudell, M. L. Chem. Rev. 1996, 96, 1179. (b) Carroll, F. I. Bioorg. Med. Chem. Lett. 2004, 14, 1889.

6. (a) Seerden, J.-P. G.; Tulp, M. Th. M.; Scheeren, H. W.; Kruse, C. G. Bioorg. Med. Chem. 1998, 6, 2103. (b) Carroll F. I.; Robinson, T. P.; Brieaddy, L. E.; Atkinson, R. N.; Mascarella, S. W.; Damaj, M. I.; Martín, B. R.; Navarro, H. A. J. Med. Chem. 2007, 50, 6383, and references cited therein.

7. (a) Brieaddy, L. E.; Mascarella, S. W.; Navarro, H. A.; Atkinson, R. N.; Damaj, M. I.; Martín, B. R.; Carroll, F. I. Tetrahedron Lett. 2001, 42, 3795. (b) Abe, H.; Arai, Y.; Aoyagi, S.; Kibayashi, C. Tetrahedron Lett. 2003, 44, 2971. (c) Wei, Z.-L.; Petukhov, P. A.; Xiao, Y.; Tückmantel, W.; George, C.; Kellar, K. J.; Kozikowski, A. P. J. Med. Chem. 2003, 46, 921.

8. Gómez-Sánchez, E.; Marco-Contelles, J. Lett. Org. Chem. 2006, 3, 827 .

9. Gómez-Sánchez, E.; Soriano, E.; Marco-Contelles, J. J. Org. Chem. 2007, 72, 8656.

10. Gómez-Sánchez, E.; Marco-Contelles, J. Tetrahedron 2005, 61, 1207.

11. Kapferer, P.; Vasella, A. Helv. Chim. Acta 2004, 87, 2764.

12. Gómez-Sánchez, E.; Soriano, E.; Marco-Contelles, J. J. Org. Chem. 2008, 73, 6784.

13. Underwood, G. R.; Friedman, H. S. J. Am. Chem. Soc. 1977, 99, 27.

14. (a) Giese, B., Jay, K. Chem. Ber. 1979, 112, 298. (b) Srikrishna, A.; Viswajanani, R.; Reddy, T. J.; Vijaykumar, D.; Kumar, P. P. J. Org. Chem. 1997, 62, 5232; (c) Liaw, D.-J.; Huang, C.-C.; Ju, J.-Y. J. Polym. Sci.Part A, 2006, 44, 3382.

15. Boivin, J.; da Silva, E.; Ourisson, G.; Zard, S. Tetrahedron Lett. 1990, 31, 2501.

16. Takasu, K.; Mizutani, S.; Nogushi, M.; Makita, K.; Ihara, M. Org. Lett. 1999, 1, 391.

17. Fraser, R. R.; Swingle, R. B. Can. J. Chem. 1970, 48, 2065.

18. Armstrong, A.; Bhonoah, Y.; Shanahan, S. E. J. Org. Chem. 2007, 72, 8019.

19. M. Legraverend, H. Boumchita, E. Bisagni, J. Heterocycl. Chem. 1990, 27, 1801. 
20. Sun, C.; Zhang, X.; Huang, H.; Zhou, P. Bioorg. Med. Chem. 2006, 14, 8574.

21. Vankar, Y.D.; Kumaravel, G.; Rao, C.T. Synth. Commun. 1989, 19, 2181.

22. LeBel, N.A.; Cherluck, R.M.; Curtis, E.A. Synthesis, 1973, 678.

23. Bayer, A.; Hansen, L. K.; Gautun, O. R. Tetrahedron: Asymmetry 2002, 13, 2407.

24. W. Zeng, S. R. Chemler, J. Am. Chem. Soc. 2007, 129, 12948.

25. Armstrong, A.; Shanahan, S. E. Org. Lett. 2005, 7, 1335. 